\title{
A Lie-Bäcklund Approach to Equivalence and Flatness of Nonlinear Systems
}

\author{
Michel Fliess, Jean Lévine, Philippe Martin, and Pierre Rouchon
}

\begin{abstract}
In this paper, a new system equivalence relation, using the framework of differential geometry of jets and prolongations of infinite order, is studied. In this setting, two systems are said to be equivalent if any variable of one system may be expressed as a function of the variables of the other system and of a finite number of their time derivatives. This is a Lie-Bäcklund isomorphism. This quite natural, though unusual, equivalence is presented in an elementary way by the inverted pendulum and the vertical take-off and landing (VTOL) aircraft. The authors prove that, although the state dimension is not preserved, the number of input channels is kept fixed. They also prove that a Lie-Bäcklund isomorphism can be realized by an endogenous feedback, i.e., a special type of dynamic feedback. Differentially flat nonlinear systems, which were introduced by the authors in 1992 via differential algebraic techniques, are generalized here and the new notion of orbitally flat systems is defined. They correspond to systems which are equivalent to a trivial one, with time preservation or not. Trivial systems are, in turn, equivalent to any linear controllable system with the same number of inputs, and consequently flat systems are linearizable by endogenous feedback. The endogenous linearizing feedback is explicitly computed in the case of the VTOL aircraft to track given reference trajectories with stability; simulations are presented.
\end{abstract}

Index Terms - Dynamic feedback, flatness, infinite-order prolongations, Lie-Bäcklund equivalence, nonlinear systems.

\section{INTRODUCTION}

$\mathbf{T}$ HE LINKS between system equivalence, system classification, and feedback design have been clarified and widely exploited for many years. To mention but one example, issued from the now well-admitted differential geometric approach to nonlinear systems [36], [58], static-state feedbacks and diffeomorphisms provide us with a natural transformation group preserving the state manifold. Two systems are said to be equivalent with respect to this group if they can be transformed into each other by some element of the group. This approach has produced numerous interesting results including the seminal ones of Jakubczyk and Respondek [40] and Hunt

Manuscript received May 12, 1996; revised June 26, 1997 and January 28, 1998. Recommended by Associate Editor, M. DiBenedetto. This work was supported in part by the European Commission's Training and Mobility of Researchers (TMR) under Contract ERBFMRX-CT970137, the G.D.R. "Medicis" of the C.N.R.S., and the P.R.C.-G.D.R. "Automatique."

M. Fliess is with the Centre de Mathématiques de Leurs Applications, École Normalle Supérieure de Cachan, 94235 Cachan, France.

J. Lévine and P. Martin are with the Centre Automatique et Systèmes, École des Mines de Paris, 77305 Fontainebleau, France (e-mail: levine@cas.ensmp.fr).

P. Rouchon is with the Centre Automatique et Systèmes, École des Mines de Paris, 75272 Paris Cédex 06, France.

Publisher Item Identifier S 0018-9286(99)03940-9. et al. [34] on static-state feedback linearization. However, as far as dynamic feedbacks are concerned, this approach might lead to serious difficulties, as demonstrated by the following elementary example: take the simple integrator

$$
\dot{x}=u
$$

and the invertible dynamic feedback

$$
\dot{v}=u
$$

(invertible in the sense of the invertibility of the input-output mapping $u \rightarrow v$ ). It yields the system $\dot{x}=\dot{v}$. To recover the original integrator, we need to pose $u=\dot{v}$, and therefore two controls $v_{1}$ and $v_{2}$ such that $v_{1}-v_{2}=$ constant lead to the same answer, which proves that the corresponding transformation is not one-to-one and that invertible dynamic feedbacks do not form a group. Indeed, this remark did not prevent several authors from getting a lot of insight into the problem of dynamic feedback linearization (see for example [11], [12], and [37] in the second half of the 1980's and later on [57], [61], [69], and [72]), but the equivalence question, which is of a more general nature, remains partly open.

For systems that are reminiscent of Fliess's differential algebraic approach [14], [15], namely systems that can be expressed in the form $F\left(x, \dot{x}, u, \dot{u}, \cdots, u^{(r)}\right)=0$, where $u^{(r)}$ denotes the time derivative of $r$ th order of the input vector $u$ and where $F$ is a polynomial vector in all its arguments, the present authors have proposed in [22] the notion of endogenous equivalence, which circumvents the above problems and provides a natural framework to the study of system classification and linearization by a restricted class of dynamic feedbacks called endogenous. In this setting, the simplest class is made up of flat systems. As a result, differentially flat, or simply flat systems, are those systems that are equivalent, in this sense, to a system without dynamics, described by a collection of independent variables, the flat output, having the same number of components as the number of control variables. Since linear controllable systems with the same number of inputs are also proved to be equivalent, in this sense, to such systems without dynamics, dynamic feedback linearization results are deduced.

In this paper the endogenous equivalence is generalized to get rid of the system differential algebraic limitations and to be able to embrace, in particular, systems defined by a general $C^{\infty}$ vector differential equation of the form $\dot{x}=f(x, u)$. The differential-algebraic framework is now replaced by a differential geometric one (see [16], [20], [21], and [24]). A related and independent approach has also been developed 
by Pomet [61]; see also an algebraic alternative approach by Jakubczyk [38], [39]. Since dynamic feedbacks require the use of time derivatives of arbitrary order, we are naturally lead to the recent differential geometric theory of jets and prolongations of infinite order, developed, in particular, in Russia, by Vinogradov and his school [76]-[78], which is already a mainstay in several parts of mathematics and physics (see [2] and [60]).

The presentation is as elementary as possible, thanks to five introductory examples, all having the same number, two, of input channels: the vertical take-off and landing aircraft (VTOL), the VTOL with a model of the actuators, the explicit and implicit inverted pendulum, and the Huygens oscillation center. With only two of them being equivalent by diffeomorphism and static-state feedback, we demonstrate that they are all equivalent in the following generalized sense: for an arbitrary pair of the above systems, to the trajectories generated by one system, there locally corresponds the trajectories of the other in a one-to-one way, or, more precisely, all the variables of one can be expressed as a function of the variables of the other and a finite number of their time derivatives.

In the differential geometric language under consideration, this equivalence, which is perhaps unusual in control theory, is well-known in physics and often receives the name of Lie-Bäcklund isomorphism [2], [35], [80]. Moreover, as will be seen on the five introductory examples, it naturally fits with important physical considerations and confirms the fact that the classic geometric state space approach, where the state lives in a fixed finite-dimensional manifold, and where the allowed transformations (diffeomorphic changes of coordinates and feedbacks) must leave this manifold invariant, is too restrictive for our purpose. Here, we prove that only the number of input variables is preserved by Lie-Bäcklund equivalence (see also [61]).

However, since, again, Lie-Bäcklund equivalence does not preserve the state dimension, it can be used to decrease the system complexity by reducing both the number of variables and equations. This particularly important property is extensively used all along this paper to extract a minimal set of relations (couplings) between a minimal set of variables that contain all the original informations on the system trajectories.

In this setting, a system is said to be differentially flat if it is Lie-Bäcklund equivalent to a trivial system, i.e., a system without dynamics, or, in other words, described by a set of independent scalar variables $\left\{y_{1}, \cdots, y_{m}\right\}$ for which no relations exist between their derivatives of any order, $m$ being the number of inputs. This set of variables is called a flat output. A flat system is also proved to be equivalent to any linear controllable system (of any dimension) with the same number of inputs. We also generalize this definition to include time scalings by introducing the notions of orbital equivalence and orbital flatness. Differentially flat and orbitally flat systems are proved to satisfy the strong accessibility property due to Sussmann and Jurdjevic [74].

Our main result explains the relationships between Lie-Bäcklund isomorphisms, endogenous feedbacks, and the classic setting of dynamic state feedback. One of the main consequences of this result is a constructive method to compute the dynamic feedback that exactly linearizes a flat system.

In spite of the tight links relating flatness and dynamic feedback linearization, the two concepts are indeed distinct. Following [50], we may argue that, though any flat system can be feedback linearized using endogenous dynamic feedbacks, flatness is a property of a system, and more precisely of its trajectories. It does not imply that one intends to then transform the system, via a dynamic feedback and appropriate changes of coordinates, to a single linear system. When a system is flat it is an indication that the nonlinear structure of the system is well characterized and one can exploit that structure in the control design. Let us stress that some of the most useful properties of flatness and flat outputs concern the system trajectories and find an immediate application in trajectory generation and tracking. Precisely, according to the flat output properties, system trajectories exactly joining a collection of points with given velocities, accelerations, jerks, etc., are easily generated (see [22]), thus replacing difficult dynamical computations by static interpolation techniques. Moreover, a tracking architecture for which the controller is fixed once and for all, independently of the particular trajectory to follow, may be obtained with stability (see [16]). Finally, let us mention the major difficulties to obtain computable criteria for checking flatness. Note that, in this perspective, the fundamental results of [34], [40], and [73] may be seen as the first sufficient conditions for checking a special kind of flatness. Nevertheless, a large number of engineering examples from many fields stemming from mechanics to chemical or biological processes, enhance the authors' belief that, since flat outputs contain all the required dynamical informations to run the system, they may often be found by inspection among the key physical variables. This feature is particularly evident on the VTOL example (see [31], [45], and [49]), whose flat output is made up with the coordinates of the Huygens oscillation center and in the numerous applications to food engineering processes [5], car equipment design [6], car parking [16], [22], [67], crane control [22], [27], [44], tracking observers [28], magnetic bearings control [43], aircraft [48], [49], and helicopter control [55], induction motors [53], chemical reactors [64], [65], etc., recently treated by this approach.

This point of view is here enhanced by sketching both the motion planning and the feedback design of the VTOL example, and some simulations showing the aircraft closedloop behavior in the presence of errors are presented.

Our paper is organized as follows. We first describe carefully our five examples. The calculations for showing their equivalence are presented in order to familiarize the reader with the infinite-dimensional differential geometric language. The second section introduces our infinite-dimensional geometry by associating to a "classic" dynamics $\dot{x}=F(x, u)$ a manifold with local coordinates the components of $x$ and the time derivatives of any order of the components of $u$. The formalism is developed in such a way that it encompasses nonlinear control systems which are not a priori written in the above form, but as a mixture of differential and nondifferential, i.e., implicit equations (remember that such a situation often occurs in practice; see, e.g., [7], [22], [27], and [54]). The 


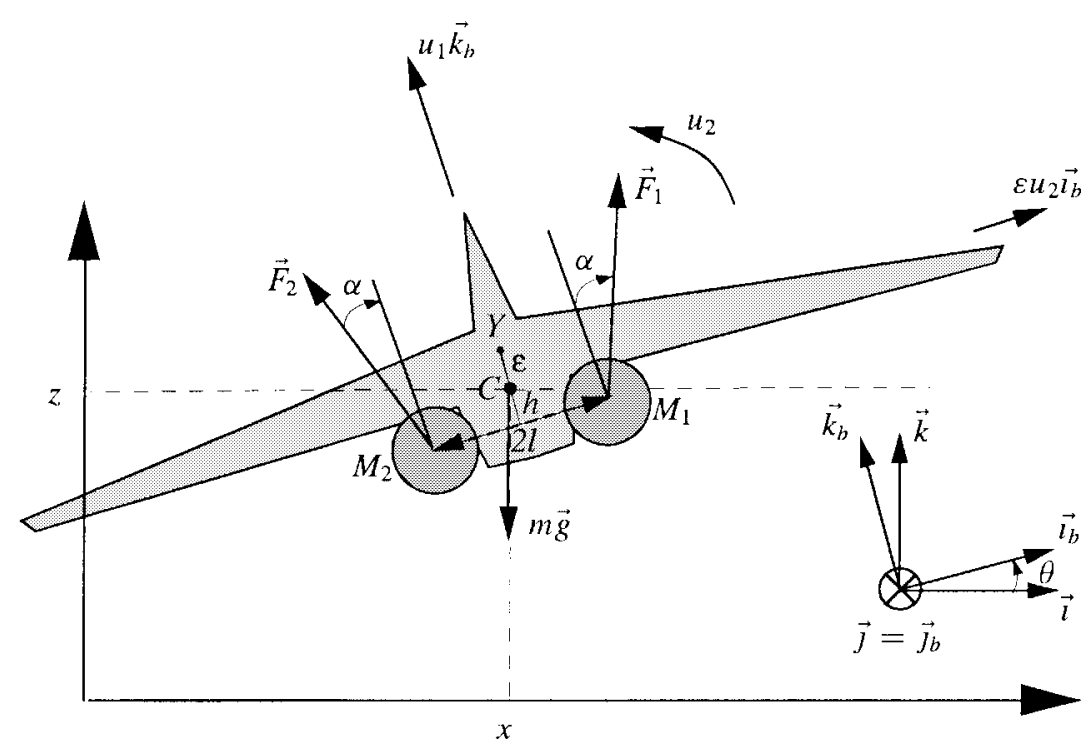

Fig. 1. The VTOL aircraft in the vertical plane.

equivalence we have in mind then becomes in this setting a change of (infinite-dimensional) local coordinates. Trivial systems and linear controllable systems are then introduced and flatness is defined. We also introduce orbital equivalence and orbital flatness where time-scaling is allowed. Various properties of this equivalence such as the invariance of the number of inputs, are proved. The strong accessibility property of a flat system is then deduced. The last section establishes the connection between Lie-Bäcklund isomorphisms and endogenous feedbacks, a special type of dynamic state feedbacks. The controller and simulations of the VTOL example are then presented, followed by a conclusion.

\section{Five InTRODUCTORY EXAMPLES}

\section{A. The VTOL Example}

We consider, as in [31], a very simplified VTOL aircraft whose evolution is restricted in the vertical plane (see Fig. 1 and the notations therein). Let $(\vec{\imath}, \vec{\jmath}, \vec{k})$ be a fixed inertial frame and $\left(\vec{\imath}_{b}, \vec{\jmath} b, \vec{k}_{b}\right)$ be a moving frame attached to the aircraft (body axes). We denote by $\theta$ the angle between the moving frame and the inertial one.

The forces acting on the system are

$$
\begin{aligned}
\vec{F}_{1} & =\left(\cos \alpha \vec{k}_{b}+\sin \alpha \overrightarrow{\imath_{b}}\right) F_{1} \\
\vec{F}_{2} & =\left(\cos \alpha \vec{k}_{b}-\sin \alpha \overrightarrow{\imath_{b}}\right) F_{2} \\
m \vec{g} & =-m g \vec{k}
\end{aligned}
$$

where $\alpha$ is a fixed angle. The weight $m \vec{g}$ is applied at the center of mass $C$; the thrusts $\vec{F}_{1}$ and $\vec{F}_{2}$ are, respectively, applied at the points $M_{1}$ and $M_{2}$, where

$$
C \vec{M}_{1}=l \overrightarrow{\imath_{b}}-h \vec{k}_{b}, \quad C \vec{M}_{2}=-l \vec{\imath}_{b}-h \vec{k}_{b}
$$

The equations of motion written in terms of the center of mass $C$ are then

$$
\begin{aligned}
m \dot{\vec{V}}_{C} & =\vec{F}_{1}+\vec{F}_{2}+m \vec{g} \\
\dot{\vec{\sigma}}_{C} & =C \vec{M}_{1} \wedge \vec{F}_{1}+C \vec{M}_{2} \wedge \vec{F}_{2}
\end{aligned}
$$

where $\vec{V}_{C}$ is the velocity of $C$ and $\vec{\sigma}_{C}$ is the angular momentum about $C$. Projecting these equations in the fixed axes, we get

$$
\begin{aligned}
m \ddot{x}_{C}= & \left(F_{1}+F_{2}\right) \cos \alpha \sin \theta+\left(F_{1}-F_{2}\right) \sin \alpha \cos \theta \\
m \ddot{z}_{C}= & \left(F_{1}+F_{2}\right) \cos \alpha \cos \theta+\left(F_{2}-F_{1}\right) \sin \alpha \sin \theta \\
& -m g \\
J \ddot{\theta}= & \left(F_{2}-F_{1}\right)(l \cos \alpha+h \sin \alpha) .
\end{aligned}
$$

Setting

$$
\begin{aligned}
\varepsilon & =\left(\frac{\sin \alpha}{l \cos \alpha+h \sin \alpha}\right) \frac{J}{m g} \\
u_{1} & =\frac{\cos \alpha}{m g}\left(F_{1}+F_{2}\right) \\
u_{2} & =\frac{l \cos \alpha+h \sin \alpha}{J}\left(F_{2}-F_{1}\right) \\
x & =\frac{x_{C}}{g} \\
z & =\frac{z_{C}}{g}
\end{aligned}
$$

the equations of motion finally read

$$
\left\{\begin{array}{l}
\ddot{x}=u_{1} \sin \theta-\varepsilon u_{2} \cos \theta \\
\ddot{z}=u_{1} \cos \theta+\varepsilon u_{2} \sin \theta-1 \\
\ddot{\theta}=u_{2} .
\end{array}\right.
$$

The dimensionless parameter $\varepsilon$ will in general be small because the angle $\alpha$ is small by construction. The normalized lengths $x$ and $z$ represent actual lengths divided by the intensity $g$ of the gravitation field, hence a normalized length of 1 represents an actual length of about $10 \mathrm{~m}$. 
The two control variables $u_{1}$ and $u_{2}$ are proportional to the vertical acceleration and torque, respectively, applied to the center of mass. From now on, (1) will be referred to as the VTOL model.

\section{B. The VTOL with a Model of the Actuators}

We now add to (1) a model of the actuators, namely differential equations that describe the way the acceleration and torque are produced

$$
\left\{\begin{array}{l}
\ddot{x}=u_{1} \sin \theta-\varepsilon u_{2} \cos \theta \\
\ddot{z}=u_{1} \cos \theta+\varepsilon u_{2} \sin \theta-1 \\
\ddot{\theta}=u_{2} \\
\dot{u}_{1}=\gamma_{1}\left(x, \dot{x}, z, \dot{z}, \theta, \dot{\theta}, u_{1}, u_{2}, v_{1}, v_{2}\right) \\
\dot{u}_{2}=\gamma_{2}\left(x, \dot{x}, z, \dot{z}, \theta, \dot{\theta}, u_{1}, u_{2}, v_{1}, v_{2}\right) .
\end{array}\right.
$$

The smooth functions $\gamma_{i}, i=1,2$, which need not be specified here, are such that the mapping $\left(v_{1}, v_{2}\right) \mapsto \gamma(x, \dot{x}, z, \dot{z}, \theta$, $\left.\dot{\theta}, u_{1}, u_{2}, v_{1}, v_{2}\right)$ is invertible for every $\left(x, \dot{x}, z, \dot{z}, \theta, \dot{\theta}, u_{1}\right.$, $u_{2}$ ) where the vector-valued function $\gamma$ stands for the vector $\left(\gamma_{1}, \gamma_{2}\right)^{T}$.

According to this invertibility, and since the variables used to describe (2) can be expressed in terms of the variables of the VTOL system and a finite number of their derivatives, it seems natural to call this new extended system equivalent to (1), though its state dimension is different.

Clearly, to every integral curve $\mathcal{C}$ of (1), defined by

$$
t \mapsto \mathcal{C}(t)=\left(x(t), \dot{x}(t), z(t), \dot{z}(t), \theta(t), \dot{\theta}(t), u_{1}(t), u_{2}(t)\right)
$$

for $t$ in an open interval $I$ of $\mathbb{R}$, there corresponds an integral curve $\tilde{\mathcal{C}}$ of (2), defined, on the interval $I$, by $t \mapsto \tilde{\mathcal{C}}(t)=(\tilde{x}(t)$, $\left.\dot{\tilde{x}}(t), \tilde{z}(t), \dot{\tilde{z}}(t), \tilde{\theta}(t), \dot{\tilde{\theta}}(t), \tilde{u}_{1}(t), \tilde{u}_{2}(t), v_{1}(t), v_{2}(t)\right)$

$$
\begin{aligned}
& \tilde{x}(t)=x(t), \quad \dot{\tilde{x}}(t)=\dot{x}(t) \\
& z(t)=z(t), \quad \dot{\tilde{z}}(t)=\dot{z}(t) \\
& \tilde{\theta}(t)=\theta(t), \quad \dot{\tilde{\theta}}(t)=\dot{\theta}(t) \\
& \tilde{u}_{1}(t)=u_{1}(t), \quad \tilde{u}_{2}(t)=u_{2}(t)
\end{aligned}
$$

and

$$
\begin{gathered}
\left(\begin{array}{l}
v_{1}(t) \\
v_{2}(t)
\end{array}\right)=\gamma^{-1}(x(t), \dot{x}(t), z(t), \dot{z}(t), \theta(t), \dot{\theta}(t), \\
\left.u_{1}(t), \dot{u}_{1}(t), u_{2}(t), \dot{u}_{2}(t)\right) .
\end{gathered}
$$

We have thus constructed a mapping $\Phi$ sending the integral curves $\mathcal{C}$ on the integral curves $\tilde{\mathcal{C}}$. Moreover, $\Phi$ preserves tangent vectors since to the tangent vector $(d / d t) \mathcal{C}(t)$ there corresponds the tangent vector $(d / d t) \tilde{\mathcal{C}}(t)$.

Conversely, it is immediate to check that $\Phi$ is invertible: every point of the integral curve $\mathcal{C}$ can be uniquely expressed as a function of $\tilde{\mathcal{C}}$. This new (inverse) mapping also preserves tangent vectors. It results that the trajectories of (1) and (2) are two different descriptions of the same object. We thus have the right to declare that (1) and (2) are equivalent.

Since the above trajectories live on manifolds with different dimensions, and since their state representations are both strongly accessible (see, e.g., [36] and [58]), this equivalence relation is indeed an unusual one. Moreover, it may involve derivatives of the input coordinates [see (4)]. The above invertible mapping $\Phi$ is called a Lie-Bäcklund isomorphism, or, sometimes, a Lie-Bäcklund transformation, and the associated equivalence relation, a Lie-Bäcklund equivalence (see Section III-B below). Note that since the model of the actuators can be realized from (1) by the invertible dynamic feedback

$$
\left\{\begin{array}{l}
\dot{u}_{1}=\gamma_{1}\left(x, \dot{x}, z, \dot{z}, \theta, \dot{\theta}, u_{1}, u_{2}, v_{1}, v_{2}\right) \\
\dot{u}_{2}=\gamma_{2}\left(x, \dot{x}, z, \dot{z}, \theta, \dot{\theta}, u_{1}, u_{2}, v_{1}, v_{2}\right)
\end{array}\right.
$$

the above equivalence relation can be interpreted in terms of dynamic feedback. Precise statements concerning this aspect may be found in Sections III-B and III-C. We will see later on that the VTOL is flat, or, in other words, equivalent to a controllable linear system. Therefore, (2) is also flat by the transitivity of the equivalence relation.

It is interesting to remark that this equivalence concept is strongly related to Cartan's absolute equivalence, as noted by Shadwick [69], and then Sluis [71], and Murray and his colleagues [57], by interpreting in the context of controlled systems the original work of Cartan [8], [9] (see [60] for a recent and nice book on this subject).

Remark also that if, in place of a model of actuators of the first order, we had a higher order dynamic extension, using only the system variables and a finite number of their time derivatives, the same result would still hold true, with the Lie-Bäcklund isomorphism $\Phi$ involving higher order derivatives of $u_{1}$ and $u_{2}$. Therefore, it may be more convenient to define such mappings as ranging the manifold with coordinates

$$
\left(t, x, \dot{x}, z, \dot{z}, \theta, \dot{\theta}, u_{1}, u_{2}, \dot{u}_{1}, \dot{u}_{2}, \cdots, u_{1}^{(k)}, u_{2}^{(k)}, \cdots\right)
$$

into itself. This implies in turn that all the notions of systems, manifolds, vector fields, etc. may also be expressed in this setting. This is precisely what we have in mind.

\section{The Inverted Pendulum Example}

We consider an inverted pendulum in the $(x, z)$-plane (see [22] and [45]), of mass $m$, controlled by means of an exterior force $\vec{F}$ applied to the point $A$ located at a distance $d$ from the center of mass $C$ of the pendulum (see Fig. 2). Let ( $\vec{\imath}$, $\vec{\jmath}, \vec{k})$ be an inertial frame. The force $\vec{F}$ reads in this frame $\vec{F}=F_{x} \vec{\imath}+F_{z} \vec{k}$. The force applied to the center of mass is the sum of $\vec{F}$ and the gravitational field: $F_{x} \vec{\imath}+\left(F_{z}-m g\right) \vec{k}$. Denote by $\left(x_{C}, O, z_{C}\right)$ the coordinates of $C$. We have

$$
m \ddot{x}_{C}=F_{x}, \quad m \ddot{z}_{C}=F_{z}-m g .
$$

Denote by $\theta$ the angle between the pendulum and the vertical axis (parallel to $\vec{k}$ ) and $J$ the inertia of the pendulum. The vector $\overrightarrow{C A}$ thus reads $\overrightarrow{C A}=-d \sin \theta \vec{\imath}-d \cos \theta \vec{k}$ and the angular momentum about $C$ is given by

$$
J \ddot{\theta} \vec{\jmath}=\overrightarrow{C A} \wedge \vec{F}=d\left(F_{z} \sin \theta-F_{x} \cos \theta\right) \vec{\jmath} .
$$


We have thus obtained

$$
\left\{\begin{array}{l}
m \ddot{x}_{C}=F_{x} \\
m \ddot{z}_{C}=F_{z}-m g \\
\frac{J}{d} \ddot{\theta}=F_{z} \sin \theta-F_{x} \cos \theta .
\end{array}\right.
$$

Setting

$$
\begin{aligned}
x & =\frac{x_{C}}{g}, \quad z=\frac{z_{C}}{g}, \quad w_{1}=\frac{F_{x}}{m g} \\
w_{2} & =\frac{F_{z}}{m g}-1, \quad \varepsilon=\frac{J}{m g d}
\end{aligned}
$$

yields

$$
\left\{\begin{array}{l}
\ddot{x}=w_{1} \\
\ddot{z}=w_{2} \\
\varepsilon \ddot{\theta}=-w_{1} \cos \theta+\left(w_{2}+1\right) \sin \theta
\end{array}\right.
$$

where $w_{1}$ and $w_{2}$ are the components of the input vector. We call this system the inverted pendulum model.

As with the previous example, it is easily seen that the normalized pendulum dynamics and the VTOL dynamics are equivalent in the sense that to every trajectory of (1), with its associated tangent vector field, there corresponds a unique trajectory of (6) with its associated tangent vector field, and conversely. This results from the formulas

$$
\left\{\begin{array}{l}
w_{1}=u_{1} \sin \theta-\varepsilon u_{2} \cos \theta \\
w_{2}=u_{1} \cos \theta+\varepsilon u_{2} \sin \theta-1
\end{array}\right.
$$

and

$$
\left\{\begin{array}{l}
u_{1}=w_{1} \sin \theta+\left(w_{2}+1\right) \cos \theta \\
u_{2}=\frac{1}{\varepsilon}\left(-w_{1} \cos \theta+\left(w_{2}+1\right) \sin \theta\right)
\end{array}\right.
$$

$x, z, \theta$ being the same in both systems.

Note that since the state dimension is preserved, the above defined mapping

$$
\left(x, z, \theta, u_{1}, u_{2}\right)=\Phi\left(x, z, \theta, w_{1}, w_{2}\right)
$$

is a diffeomorphism: our notion of equivalence coincides here with the classic equivalence by diffeomorphism and static feedback (see, e.g., [36] and [58]).

\section{An Implicit Model of Pendulum}

We also consider the following variant of (6), obtained by eliminating the control variables $w_{1}$ and $w_{2}$

$$
\varepsilon \ddot{\theta}=-\ddot{x} \cos \theta+(\ddot{z}+1) \sin \theta \text {. }
$$

This is a single implicit differential equation with three unknown functions $(x, z, \theta)$. Such an implicit differential equation is thus called underdetermined. The number of degrees of freedom is 2, i.e., it is the number of input channels: by posing $\ddot{x}=w_{1}$ and $\ddot{z}=w_{2}$, the system becomes determined and the equivalence with the normalized inverted pendulum is clear. Therefore, besides the fact that the control variables are not specified, it contains exactly the same information as (6). This shows in particular that our notion of equivalence is

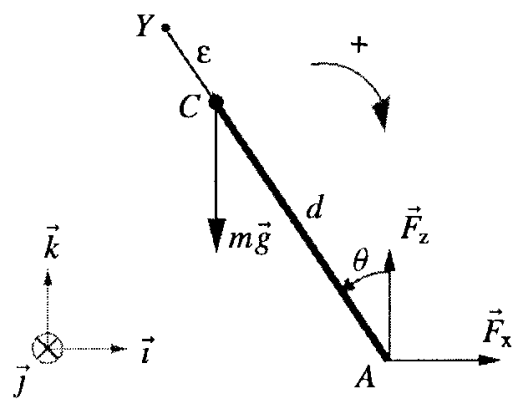

Fig. 2. The inverted pendulum in the vertical plane.

intrinsic in the sense that it does not depend on the choice of input, state, and other system variables.

The equivalence of (9) to the VTOL model (1) may be proven in the same manner by using the relations (8).

\section{E. The Huygens Oscillation Center}

Finally, we consider the following implicit differential system (see [3], [18], [19], and [45]) with four unknown functions and two equations

$$
\left\{\begin{array}{l}
\left(\xi-\nu_{1}\right)^{2}+\left(\zeta-\nu_{2}\right)^{2}=\varepsilon^{2} \\
\ddot{\xi}\left(\zeta-\nu_{2}\right)-\left(\xi-\nu_{1}\right)(\ddot{\zeta}+1)=0 .
\end{array}\right.
$$

This system admits the following geometrical interpretation. Let $(\xi, \zeta)$ and $\left(\nu_{1}, \nu_{2}\right)$ be the coordinates of two points, $Y$ and $N$, respectively, in the $(x, z)$ plane $[(\vec{\imath}, \vec{\jmath}, \vec{k})$ is the inertial frame associated to $(x, y, z)]$. The first equation simply means that the Euclidian distance $d(Y, N)$ from $Y$ to $N$ is equal to $\varepsilon$. The second equation means that the vector, sum of the acceleration of $Y$, and of the constant vector $\vec{k}$, is colinear to the vector $\overrightarrow{Y N}{ }^{1}$

$$
\frac{\ddot{\xi}}{\ddot{\zeta}+1}=\frac{\xi-\nu_{1}}{\zeta-\nu_{2}}
$$

These equations are satisfied by a single rigid body, in the vertical (normalized) gravity field $-\vec{k}$, free to turn about a horizontal axis, fixed in the body and parallel to $\vec{f} Y$ is then the center of oscillation (often called Huygens oscillation center) and $N$ the center of suspension (see [79, p. 132]).

Thus for the VTOL, $N$ coincides with $C\left(\nu_{1}=x, \nu_{2}=z\right)$ and $Y$ (see Fig. 1) corresponds to

$$
\xi=x+\varepsilon \sin \theta, \quad \zeta=z+\varepsilon \cos \theta .
$$

Similarly, for the pendulum, $N$ coincides with $A$ and $Y$ is the point belonging to the axis $A C$, corresponding to (12) (see Fig. 2).

Another property of the oscillation center is that it summarizes in a static way, i.e., without integrating any differential equation, all the information needed to recover the dynamic behavior of the rest of the solid. More precisely, once its trajectory is given, one can completely recover the trajectory

\footnotetext{
${ }^{1}$ Note that the physical properties of $Y$ are directly translated into the implicit differential system (10), whereas decoding them from an explicit statespace realization would not be that easy (see also [27] for a more detailed discussion on this subject).
} 
to be followed by the center of mass and the corresponding force to apply: from (11) and (12), we get

$$
\tan \theta=\frac{\xi-x}{\zeta-z}=\frac{\ddot{\xi}}{\ddot{\zeta}+1}
$$

or

$$
\begin{aligned}
\theta & =\arctan \left(\frac{\ddot{\xi}}{\ddot{\xi}+1}\right) \\
\sin \theta & =\frac{\ddot{\xi}}{\sqrt{(\ddot{\xi})^{2}+(\ddot{\zeta}+1)^{2}}} \\
\cos \theta & =\frac{\ddot{\zeta}+1}{\sqrt{(\ddot{\xi})^{2}+(\ddot{\zeta}+1)^{2}}} .
\end{aligned}
$$

Finally, we have

$$
\begin{aligned}
& x=\xi-\varepsilon \frac{\ddot{\xi}}{\sqrt{(\ddot{\xi})^{2}+(\ddot{\zeta}+1)^{2}}} \\
& z=\zeta-\varepsilon \frac{\ddot{\zeta}+1}{\sqrt{(\ddot{\xi})^{2}+(\ddot{\zeta}+1)^{2}}}
\end{aligned}
$$

and the force is easily deduced by twice differentiating the expressions of $x$ and $z$. This property, called differential flatness, will be studied in greater details in Section III-C.

To prove the equivalence of (10) to (6) or (1), by transitivity, it suffices to prove that (10) and (9) are equivalent. Starting from (10), differentiating twice (12), we get

$$
\begin{aligned}
& \ddot{\xi}=\ddot{x}+\varepsilon \ddot{\theta} \cos \theta-\varepsilon \dot{\theta}^{2} \sin \theta \\
& \ddot{\zeta}=\ddot{z}-\varepsilon \ddot{\theta} \sin \theta-\varepsilon \dot{\theta}^{2} \cos \theta .
\end{aligned}
$$

Taking into account the fact that $\xi-x=\varepsilon \sin \theta$ and $\zeta-z=$ $\varepsilon \cos \theta$, we have

$$
\begin{aligned}
& \ddot{\xi}(\zeta-z)-(\ddot{\zeta}+1)(\dot{\xi}-x) \\
& \quad=\varepsilon(\ddot{\xi} \cos \theta-(\ddot{\zeta}+1) \sin \theta) \\
& \quad=\varepsilon(\varepsilon \ddot{\theta}+\ddot{x} \cos \theta-(\ddot{z}+1) \sin \theta) .
\end{aligned}
$$

Therefore, if (10) holds true, then (9) does too, and conversely, which proves the equivalence.

As a consequence, all the variables of (6) or (1) can be expressed, in an invertible way, in terms of $\xi, \zeta$ and a finite number of their time derivatives $\dot{\xi}, \ddot{\xi}, \cdots, \dot{\zeta}, \ddot{\zeta}, \cdots$. Indeed, in the pendulum case, it suffices to compute $w_{1}=\ddot{x}$ and $w_{2}=\ddot{z}$ by differentiating twice the expressions of $x$ and $z$

$$
\begin{aligned}
& w_{1}=\frac{d^{2}}{d t^{2}}\left(\xi-\varepsilon \frac{\ddot{\xi}}{\sqrt{\left((\ddot{\xi})^{2}+(\ddot{\zeta}+1)^{2}\right.}}\right) \\
& w_{2}=\frac{d^{2}}{d t^{2}}\left(\zeta-\varepsilon \frac{\ddot{\zeta}+1}{\sqrt{\left((\ddot{\xi})^{2}+(\ddot{\zeta}+1)^{2}\right.}}\right)
\end{aligned}
$$

which involve derivatives of $\xi$ and $\zeta$ up to fourth order.

Accordingly, in the VTOL case, it suffices to use (8) to obtain $u_{1}$ and $u_{2}$, thus also involving derivatives of $\xi$ and $\zeta$ up to fourth order.
Comparing this example with the second one, we find that, in (10), the control variables are $\nu_{1}$ and $\nu_{2}$. Remember that $x=\nu_{1}$ and $z=\nu_{2}$ and, by (12), we have $\ddot{\nu}_{1}=w_{1}$ and $\ddot{\nu}_{2}=w_{2}$, where $\left(w_{1}, w_{2}\right)$ is the input of the pendulum model (6). Thus, (10) may be seen as a reduced model where we have removed the double integrators of the VTOL or the inverted pendulum.

To summarize, the first three examples are given by state representations with different dimensions, the first and the third example being described by six variables, whereas the second is eight-dimensional, and the last two examples are not given in classic state variable representation. However, they are all equivalent as far as we accept to deal with coordinates and a finite, but a priori unprescribed, number of their derivatives with respect to time. This is why, in the first example, we need to consider coordinates of the form

$$
\left(t, x, v_{x}, z, v_{z}, \theta, v_{\theta},\left(u_{1}^{\left(\mu_{1}\right)}\right)_{\mu_{1} \geq 0},\left(u_{2}^{\left(\mu_{2}\right)}\right)_{\mu_{2} \geq 0}\right)
$$

where $v_{x}, v_{z}$, and $v_{\theta}$ correspond to the velocity of $x, z$, and $\theta$, respectively, and where $u_{j}^{\left(\mu_{j}\right)}$ corresponds to the derivative of order $\mu_{j}$ of $u_{j}, j=1,2$, with respect to time.

The manifold associated to (1) is thus the Cartesian product

$$
\mathbb{R} \times \mathbb{R} \times \mathbb{R} \times \mathbb{R} \times \mathbb{R} \times \mathbb{S}^{1} \times \mathbb{R} \times \mathbb{R}^{\infty} \times \mathbb{R}^{\infty}
$$

A simple introduction to the basic properties of such infinitedimensional differential manifolds is provided in the next section.

\section{INFINITE-DIMENSIONAL DIFFERENTIAL GEOMETRY}

\section{A. Infinite Number of Coordinates}

1) Explicit State Variable Representations: Consider the dynamics

$$
\dot{x}=f(x, u)
$$

where $f$ is smooth on an open subset $X \times U \subset \mathbb{R}^{n} \times \mathbb{R}^{m}$, $x$ is the state, and $u$ is the control input.

The mapping $f$ is in fact an infinite collection of vector fields parameterized by $u$. More precisely, to define an integral curve of (16), we not only need to specify the initial condition $x_{0}$ at time $t=0$, but also the smooth time function $t \mapsto u(t)$ on a given time interval. This infinite-dimensional dependence on the input $u$ is not well-adapted when considering dynamic feedback. According to the examples of the preceding section, we develop a slightly different standpoint where the integral curves of (16) are described in a more compact way as smooth functions $t \mapsto(x(t), u(t))$, parameterized by initial conditions only. More precisely, we consider initial conditions in the form of the infinite sequence $\xi_{0}=\left(x_{0}, u_{0}, \dot{u}_{0}, \cdots, u_{0}^{(\mu)}, \cdots\right)$, where the derivatives of $u$ of any order at time $t=0$ are noted $u_{0}^{(\mu)}$, with $\mu \geq 0$. We are therefore led to complete the original coordinates $(x, u)$ by the infinite sequence of coordinates $\xi=\left(x, u, \dot{u}, \cdots, u^{(\mu)}, \cdots\right) \in X \times U \times \mathbb{R}_{m}^{\infty}$, where we have denoted by $\mathbb{R}_{m}^{\infty}=\mathbb{R}^{m} \times \mathbb{R}^{m} \times \cdots$, the product of a countably infinite number of copies of $\mathbb{R}^{m}$. In this context, a smooth function is a function smoothly depending on a finite 
(but arbitrary) number of coordinates. Then, if we prolong the original vector field $f$ as

$$
F(\xi)=(f(x, u), \dot{u}, \ddot{u}, \cdots)
$$

(16) reads

$$
\dot{\xi}=F(\xi)
$$

with $\xi(0)=\xi_{0}$. Therefore, (17) defines a vector field, in the classic sense, on the infinite-dimensional manifold $X \times U \times$ $\mathbb{R}_{m}^{\infty}$.

The same conclusion is reached in another way, by considering the next Lie derivative formula: take a smooth function $h$, i.e., smoothly depending on $x, u$, and a finite number $r$ of derivatives of $u$. We adopt the classic notations $(\partial h / \partial x) f=\sum_{i=1}^{n}\left(\partial h / \partial x_{i}\right) f_{i}$ and $\left(\partial h / \partial u^{(k)}\right) u^{(k+1)}=$ $\sum_{i=1}^{m}\left(\partial h / \partial u_{i}^{(k)}\right) u_{i}^{(k+1)}$. The time derivative of $h$ along a trajectory of (16) is given by

$$
\frac{d h}{d t}=\frac{\partial h}{\partial x} f+\frac{\partial h}{\partial u} \dot{u}+\cdots+\frac{\partial h}{\partial u^{(r)}} u^{(r+1)}
$$

at every point $\left(x(t), u(t), \dot{u}(t), \cdots, u^{(r)}(t), \cdots\right)$. Note that, though $h$ depends only on derivatives of $u$ up to order $r$, the coordinate $u^{(r+1)}$ is required, which is one more motivation to consider the coordinates made up with the whole sequence of derivatives of $u$.

This formula may be interpreted as the Lie derivative of $h$ with respect to the infinite-dimensional vector field

$$
\begin{aligned}
& \left(x, u, u^{(1)}, u^{(2)}, \cdots\right) \mapsto F\left(x, u, u^{(1)}, u^{(2)}, \cdots\right) \\
& \quad=\left(f(x, u), u^{(1)}, u^{(2)}, \cdots\right)
\end{aligned}
$$

or, with notations easily understood from the Lie derivative formula (18)

$$
F\left(x, u, u^{(1)}, u^{(2)}, \cdots\right)=f(x, u) \frac{\partial}{\partial x}+\sum_{\mu=0}^{\infty} u^{(\mu+1)} \frac{\partial}{\partial u^{(\mu)}} .
$$

Note that each component of $F$ is a smooth function, i.e., depends smoothly on a finite number of coordinates.

Therefore, to the controlled system (16), where $f$ is a family of vector fields parameterized by $u$, we substitute the following system definition with a "true" vector field on an infinite-dimensional manifold.

Definition 1: A classic system is a pair $\left(X \times U \times \mathbb{R}_{m}^{\infty}, F\right)$ where $F$ is a smooth vector field on $X \times U \times \mathbb{R}_{m}^{\infty}$.

Notations: All along this paper, we shall use the following notations: $u^{(0)}=u, u^{(1)}=\dot{u}, u^{(2)}=\ddot{u}, \cdots, \bar{u}^{k}=$ $\left(u^{(0)}, u^{(1)}, \cdots, u^{(k)}\right)$ for every $k$, and $\bar{u}=\left(u^{(0)}, u^{(1)}, \cdots\right)$.

Remark 1: To be rigorous we must say something of the underlying topology and differentiable structure of $\mathbb{R}_{m}^{\infty}$ to be able to speak of neighborhoods and smooth objects. This topology is the Fréchet topology (see, e.g., [30] for details), which makes things look as if we were working on the product of $k$ copies of $\mathbb{R}^{m}$ for a "large enough" $k$. For our purpose it is enough to know that a basis of the open sets of this topology consists of infinite products $U_{0} \times U_{1} \times \cdots$ of open sets $U_{i}$ of $\mathbb{R}^{m}$, all but a finite number of them being equal to $\mathbb{R}^{m}$, and that a function is smooth, or $C^{\infty}$, if it depends on a finite but arbitrary number of variables and is smooth, or $C^{\infty}$, in the usual sense. In the same way a mapping $\Phi: \mathbb{R}_{m}^{\infty} \rightarrow \mathbb{R}_{n}^{\infty}$ is smooth if all of its components are smooth functions.

Notice also that $\mathbb{R}_{m}^{\infty}$ equipped with the Fréchet topology lacks many useful properties (see also [80]); very useful and familiar theorems in finite-dimensional analysis (see, e.g., [1] and [13]) such as the implicit function theorem, Frobenius theorem, or the straightening out theorem no longer hold true.

We can also define manifolds modeled on $\mathbb{R}_{m}^{\infty}$ using the standard machinery. The reader not interested in these technicalities can safely ignore the details and will not lose much by replacing "manifold modeled on $\mathbb{R}_{m}^{\infty}$ " by "open set of $\mathbb{R}_{m}^{\infty}$."

Remark 2: We saw above how a "classic" control system of the form (16) fits into our definition. There is nevertheless an important difference: we lose the notion of state dimension (see the VTOL examples with and without a model of the actuators as another illustration of this aspect). Indeed

$$
\dot{x}=f(x, u), \quad(x, u) \in X \times U \subset \mathbb{R}^{n} \times \mathbb{R}^{m}
$$

and

$$
\begin{aligned}
& \dot{x}=f(x, u) \\
& \dot{u}=v
\end{aligned}
$$

now have the same description $\left(X \times U \times \mathbb{R}_{m}^{\infty}, F\right)$, with

$$
F\left(x, u, u^{(1)}, u^{(2)}, \cdots\right)=\left(f(x, u), u^{(1)}, u^{(2)}, \cdots\right)
$$

in our formalism: indeed the mapping $t \mapsto(x(t), u(t))$ is a trajectory of (21) if and only if the mapping $t \mapsto$ $(x(t), u(t), \dot{u}(t))$ is a trajectory of (22). This situation is not surprising since the state dimension is of course not preserved by dynamic feedback. On the other hand we will see that there is still a notion of input dimension.

Example 1: The trivial system $\left(\mathbb{R}_{m}^{\infty}, F_{m}\right)$, with coordinates $y=\left(y_{1}, \cdots, y_{m}\right), y^{(1)}=\left(y_{1}^{(1)}, \cdots, y_{m}^{(1)}\right), y^{(2)}=\left(y_{1}^{(2)}, \cdots\right.$, $\left.y_{m}^{(2)}\right), \cdots$ and vector field

$$
F_{m}\left(y, y^{(1)}, y^{(2)}, \cdots\right)=\left(y^{(1)}, y^{(2)}, \cdots\right)
$$

or with the differential operator notation

$$
F_{m}\left(y, y^{(1)}, y^{(2)}, \cdots\right)=\sum_{i=1}^{m} \sum_{\nu_{i} \geq 0} y_{i}^{\left(\nu_{i}+1\right)} \frac{\partial}{\partial y_{i}^{\left(\nu_{i}\right)}}
$$

describes any system made up of $m$ independent chains of integrators of arbitrary lengths, and in particular the direct transfer $y_{i}=u_{i}, i=1, \cdots, m$.

Example 2: A (classic) linear system with $m$ input channels is described by $\left(\mathbb{R}^{n} \times \mathbb{R}_{m}^{\infty}, L\right)$ with the infinite vector field $L$ given by

$$
L(x, \bar{u})=(A x+B u) \frac{\partial}{\partial x}+\sum_{\mu=0}^{\infty} u^{(\mu+1)} \frac{\partial}{\partial u^{(\mu)}}
$$

where $A$ is an $n \times n$ matrix and $B$ an $m \times n$ matrix. 
2) The General Case: As suggested by the implicit representations of the pendulum (9) and oscillation center (10) of Sections II-D and II-E, it may be interesting to generalize the above considerations to systems without reference to the particular state variable representation we are working with.

Considering the vector field $F$ of (20), it is made up with a drift part $f(x, u)(\partial / \partial x)$ and an infinite control part $\sum_{\mu=0}^{\infty} u^{(\mu+1)}\left(\partial / \partial u^{(\mu)}\right)$. The infinite control part may be seen as the projection of $F$ by the projection mapping $\pi: X \times U \times$ $\mathbb{R}_{m}^{\infty} \rightarrow U \times \mathbb{R}_{m}^{\infty}$, where $\pi(x, u, \dot{u}, \ddot{u}, \cdots)=(u, \dot{u}, \ddot{u}, \cdots)$. For any $\bar{u} \in U \times \mathbb{R}_{m}^{\infty}, \pi^{-1}(\bar{u})$, which may be identified with the state space $X$, is finite-dimensional; $\pi^{-1}(\bar{u})$ is called a fiber. Thus $\pi$ induces a structure of fibered manifold on the manifold $X \times U \times \mathbb{R}_{m}^{\infty}$, with finite-dimensional fibers of constant dimension (see [1] for a discussion of these notions in the finite-dimensional case). We now use this property as a guideline for a general definition. A submersion is a mapping which, in suitable local coordinates, is a projection.

We consider a pair $(\mathcal{M}, F)$ where $\mathcal{M}$ is a smooth manifold-possibly of infinite-dimension-and $F$ is a smooth vector field on $\mathcal{M}$. This pair does not generally define a system in a satisfactory way since, for instance, the number of input channels may be infinite. Further assumptions are needed to guarantee that $F$ depends only on a finite number of independent input channels, as in the classic case.

Definition 2: We say that the pair $(\mathcal{M}, F)$ is a system if, and only if, there exists a smooth submersion $\pi$ to the trivial system $\left(\mathbb{R}_{m}^{\infty}, F_{m}\right)^{2}$ with global coordinates $\bar{u}=(u, \dot{u}, \ddot{u}, \cdots)$, such that every fiber $\zeta=\pi^{-1}(\bar{u})$ is finite-dimensional with locally constant dimension for every $\bar{u}$.

As a consequence of the definition, a local system of coordinates is given by $\xi=(\zeta, \bar{u})$, with $\zeta \in \mathbb{R}^{n}$ for some $n$, and with the $m$-tuple $u$ playing the role of $m$ independent input channels. A control system locally looks like an open subset of $\mathbb{R}^{n} \times \mathbb{R}_{m}^{\infty}$ with vector field

$$
F(\xi)=g(\zeta, \bar{u}) \frac{\partial}{\partial \zeta}+\sum_{\nu \geq 0} u^{(\nu+1)} \frac{\partial}{\partial u^{(\nu)}}
$$

where all the components of $g$ depend only on a finite number of coordinates. A trajectory of the system is a mapping $t \mapsto$ $\xi(t)=(\zeta(t), \bar{u}(t))$ such that $\dot{\xi}(t)=F(\xi(t))$, or equivalently $\dot{\zeta}=g(\zeta, \bar{u})$. Note that the existence of a local solution to this differential equation is once more implied by the fact that $g$ depends only on a finite number of coordinates, where the classic local existence result on finite-dimensional manifolds apply.

Note that our definition of system does not distinguish between state and input variables since they are both deduced from the submersion $\pi$ which is assumed to exist, but not fixed a priori.

Example 3: For the classic system $\left(X \times U \times \mathbb{R}_{m}^{\infty}, F\right)$ with local coordinates $\xi=(x, \bar{u})=(x, u, \dot{u}, \ddot{u}, \cdots)$ and

$$
F(\xi)=f(x, u) \frac{\partial}{\partial x}+\sum_{\nu \geq 0} u^{(\nu+1)} \frac{\partial}{\partial u^{(\nu)}}
$$

\footnotetext{
${ }^{2}$ See Example 1.
}

the submersion $\pi$ is just the projection $(x, \bar{u}) \mapsto \bar{u}$ from $X \times U \times \mathbb{R}_{m}^{\infty}$ to the trivial system $\left(\mathbb{R}_{m}^{\infty}, F_{m}\right)$ with $F_{m}=$ $\sum_{\nu>0} u^{(\nu+1)}\left(\partial / \partial u^{(\nu)}\right)$.

Example 4: In the coordinates $\left(x, v_{x}, z, v_{z}, \theta, v_{\theta}, u_{1}^{\left(\mu_{1}\right)}\right.$, $\left.u_{2}^{\left(\mu_{2}\right)} ; \mu_{1}, \mu_{2} \geq 0\right)$, the vector field associated to the system (1) is given by

$$
\begin{aligned}
F= & v_{x} \frac{\partial}{\partial x}+\left(u_{1} \sin \theta-\varepsilon u_{2} \cos \theta\right) \frac{\partial}{\partial v_{x}}+v_{z} \frac{\partial}{\partial z} \\
& +\left(u_{1} \cos \theta+\varepsilon u_{2} \sin \theta-1\right) \frac{\partial}{\partial v_{z}}+v_{\theta} \frac{\partial}{\partial \theta}+u_{2} \frac{\partial}{\partial v_{\theta}} \\
& +\sum_{\mu_{1} \geq 0} u_{1}^{\left(\mu_{1}+1\right)} \frac{\partial}{\partial u_{1}^{\left(\mu_{1}\right)}}+\sum_{\mu_{2} \geq 0} u_{2}^{\left(\mu_{2}+1\right)} \frac{\partial}{\partial u_{2}^{\left(\mu_{2}\right)}} .
\end{aligned}
$$

Similar expressions can be easily obtained for the vector fields corresponding to (2) or (6), which correspond to a different choice of submersion. The vector field associated to (9), in the coordinates $\left(\theta, v_{\theta}, x, \dot{x}, \ddot{x}, \cdots, z, \dot{z}, \ddot{z}, \cdots\right)$, is

$$
\begin{aligned}
& v_{\theta} \frac{\partial}{\partial \theta}+\frac{1}{\varepsilon}(-\ddot{x} \cos \theta+(\ddot{z}+1) \sin \theta) \frac{\partial}{\partial v_{\theta}} \\
&+\sum_{\mu=0}^{\infty} x^{(\mu+1)} \frac{\partial}{\partial x^{(\mu)}}+\sum_{\mu=0}^{\infty} z^{(\mu+1)} \frac{\partial}{\partial z^{(\mu)}} .
\end{aligned}
$$

As we have seen in the first section, the integral curves of (26) and (27) are transformed into each other by a smooth mapping and the respective tangent vectors are transformed accordingly. Therefore, the same transformation should change the vector field (26) into (27). The underlying equivalence relation will be precised in the next section.

Remark 3: Our definition of a system is adapted from the notion of diffiety, due to Vinogradov (see, e.g., [76]), which deals with some questions on systems of (partial) differential equations. By definition, a diffiety is a pair $(\mathcal{M}, C T \mathcal{M})$ (see, also, [80]), where $\mathcal{M}$ is a smooth manifold-possibly of infinite-dimension - and $C T \mathcal{M}$ is a Cartan distribution on $\mathcal{M}$, namely an involutive finite-dimensional distribution on $\mathcal{M}$. Remember that involutive means that the Lie bracket of any two vector fields of $C T \mathcal{M}$ is itself in $C T \mathcal{M}$ (see, e.g., [1]). As we are only working with systems with lumped parameters, hence governed by ordinary differential equations, we consider diffieties with one-dimensional Cartan distributions, which are thus always involutive.

A similar definition of a control system by an infinite vector field (18) is also introduced in [61].

Until now, we have chosen to single out a particular vector field rather than work with the one-dimensional distribution it spans. The difference is simply explained in terms of time scaling: the distribution spanned by the vector field $F$, noted $\operatorname{span}(F)$, is made of vector fields of the form $F_{\gamma} \triangleq \gamma \cdot F$ where $\gamma$ is a smooth function on $\mathcal{M}$. Therefore, changing $F$ into $F_{\gamma}$ can be interpreted as the time-scaling $d \tau / d t=1 / \gamma$ in a neighborhood of a point where $\gamma \neq 0$. Note that this time change may depend on a finite number of components of $\xi$. Indeed, $d \xi / d \tau=\gamma(\xi) F(\xi)$ becomes $d \xi / d t=F(\xi)$. Though not always necessary, it is often useful to introduce an additional coordinate, corresponding to time, to deal with such scalings and the original manifold is thus replaced by 
$\mathbb{R} \times \mathcal{M}$ with coordinates $(t, \xi)$. The vector field $F$ is, in this case, replaced by $(\partial / \partial t)+F$. Thus, since $\operatorname{span}((\partial / \partial t)+$ $F)=\left\{\gamma((\partial / \partial t)+F) \mid \gamma \in C^{\infty}(\mathbb{R} \times \mathcal{M}), \gamma \neq 0\right\}$ is a one-dimensional distribution, denoting by $\tau$ a local basis, the diffiety $(\mathbb{R} \times \mathcal{M}, \operatorname{span}((\partial / \partial t)+F))$ corresponds to the differential system $d t / d \tau=\gamma, d x / d \tau=\gamma F$, which is equivalent to $d x / d t=F, \gamma$ corresponding, as before, to the time-scaling. Therefore, the above definition can be slightly generalized, to cope with time-varying systems, by saying that a system is a diffiety $\mathbb{R} \times \mathcal{M}$, endowed with a one-dimensional distribution and a submersion $\pi$ to the trivial diffiety $\mathbb{R} \times \mathbb{R}_{m}^{\infty}$, inducing a fibration of $\mathbb{R} \times \mathcal{M}$ with finite-dimensional fibers of locally constant dimension.

Remark 4: In place of the distribution spanned by our vector field $F$, we could have worked with codistributions (cf. [1]), as already done in nonlinear control theory by several authors (see, e.g., [29]). On the manifold $\mathcal{M}$ with coordinates $\left(t, x, u, u^{(1)}, \cdots\right)$, we can indeed define a 1 -form $\omega$ as a finite linear combination of $\left\{d t, d x_{i}, d u_{j}^{(\mu)} \mid i=1, \cdots, n\right.$; $j=1, \cdots, m ; \mu \geq 0\}$. The finiteness requirement is implied by the fact that, by definition, a smooth function, and therefore its differential, depends only upon a finite number of variables. The codistribution orthogonal to the vector field $F$ is spanned by the 1 -forms $\omega$ such that $\langle F, \omega\rangle=0$. If $F$ is defined by (20), its orthogonal codistribution is spanned by the infinite set of 1-forms, often called Cartan 1-forms (see, e.g., [68] and [80])

$$
\begin{aligned}
& \left\{d x_{i}-f_{i}(x, u) d t, d u_{j}^{(\mu)}-u_{j}^{(\mu+1)} d t \mid i=1, \cdots, n ;\right. \\
& \quad j=1, \cdots, m ; \mu \geq 0\} .
\end{aligned}
$$

A thorough analysis of this setting has been given by van Nieuwstadt et al. [57].

\section{B. Changes of Coordinates, Lie-Bäcklund Mappings, and System Equivalence}

In this section we define an equivalence relation formalizing the idea that two systems are "equivalent" if there is an invertible transformation exchanging their trajectories. As we will see later, the relevance of this rather natural equivalence notion lies in the fact that it admits an interpretation in terms of dynamic feedback.

Consider two systems $(\mathcal{M}, F)$ and $(\mathcal{N}, G)$ and a smooth mapping $\Phi: \mathcal{M} \rightarrow \mathcal{N}$ (remember that by definition every component of a smooth mapping depends only on a finite number of coordinates). Let $p \in \mathcal{M}$ and denote by $q=\Phi(p)$.

If $t \mapsto \xi(t)$ is a trajectory of $(\mathcal{M}, F)$ in a neighborhood of $p$, i.e.,

$$
\forall t, \quad \dot{\xi}(t)=F(\xi(t))
$$

the composed mapping $t \mapsto \zeta(t)=\Phi(\xi(t))$ remains in a neighborhood of $q$ and satisfies the chain rule

$$
\dot{\zeta}(t)=\frac{\partial \Phi}{\partial \xi}(\xi(t)) \cdot \dot{\xi}(t)=\frac{\partial \Phi}{\partial \xi}(\xi(t)) \cdot F(\xi(t)) .
$$

We insist that the above expressions involve only finite sums even if the matrices and vectors have infinite sizes: indeed a row of $\partial \Phi / \partial \xi$ contains only a finite number of nonzero terms because a component of $\Phi$ depends only on a finite number of coordinates.

Now if the vector fields $F$ and $G$ are $\Phi$-related (cf. [1]) at $(p, q)$, i.e.,

$$
G(\Phi(\xi))=\frac{\partial \Phi}{\partial \xi}(\xi) . F(\xi)
$$

for all $\xi$ in a neighborhood of $p$, then

$$
\dot{\zeta}(t)=G(\Phi(\xi(t)))=G(\zeta(t))
$$

which means that $t \mapsto \zeta(t)=\Phi(\xi(t))$ is a trajectory of $(\mathcal{N}, G)$. If, moreover, $\Phi$ has a smooth inverse $\Psi$ then obviously $G, F$ are also $\Psi$-related at $(q, p)$, and there is a local one-to-one correspondence between the trajectories of the two systems. We call such an invertible $\Phi$ relating $F$ and $G$ at the pair $(p, q)$ an endogenous transformation at $(p, q)$, which means that the original (endogenous) variables of the system are transformed without creation of new exogeneous variables.

This definition can be extended to the time-varying case and time-scaling, with endogenous transformations replaced by the more general notion of Lie-Bäcklund isomorphisms. To this aim, we denote by (span $F$ ) [respectively, (span $G$ )] the one-dimensional distribution generated by the vector field $F$ (respectively, $G$ ).

Definition 3: The mapping $\Phi$ is said to be a Lie-Bäcklund isomorphism from $[\mathcal{M}$, $(\operatorname{span} F)]$ to $[\mathcal{N},(\operatorname{span} G)]$ at the pair of points $(p, q)$ with $p \in \mathcal{M}$ and $q=\Phi(p) \in \mathcal{N}$, if $\Phi$ is a smooth mapping from a neighborhood of $p$ in $\mathcal{M}$ to a neighborhood of $q$ in $\mathcal{N}$, preserving the distributions $(\operatorname{span} F)$ and (span $G)$, namely such that its tangent mapping $T \Phi$ locally satisfies $T \Phi(\operatorname{span}(F))=\operatorname{span}(G)$, and if it has a smooth inverse $\Psi$ from a neighborhood of $q$ in $\mathcal{N}$ to a neighborhood of $p$ in $\mathcal{M}$ with $T \Psi(\operatorname{span}(G))=\operatorname{span}(F)$.

Clearly, $\Phi$ is Lie-Bäcklund if, and only if, it locally relates $F$ to an element $\eta G$ of the distribution $\operatorname{span}(G)$ and if its inverse $\Psi$ relates $G$ to an element $\gamma F$ of $\operatorname{span}(F)$. A Lie-Bäcklund isomorphism is thus an endogenous transformation if $\gamma \equiv \eta \equiv 1$, which means that the time is left unchanged.

Endogenous transformations naturally lead to the following concept of differential equivalence.

Definition 4: Two systems $(\mathcal{M}, F)$ and $(\mathcal{N}, G)$ are differentially equivalent at $(p, q) \in \mathcal{M} \times \mathcal{N}$, or shortly, equivalent at $(p, q)$, if there exists a smooth mapping $\Phi$ from a neighborhood of $p$ to a neighborhood of $q=\Phi(p)$ which is an endogenous transformation at $(p, q)$.

$(\mathcal{M}, F)$ and $(\mathcal{N}, G)$ are (differentially) equivalent if there exists a smooth mapping $\Phi$ from an open dense subset $\mathcal{D} \subset \mathcal{M}$ to $\mathcal{N}$ which is an endogenous transformation from $(\mathcal{M}, F)$ to $(\mathcal{N}, G)$ in a neighborhood of every pair of points $(p, \Phi(p))$, for $p$ in $\mathcal{D}$.

This (differential geometric) notion of equivalence corresponds to the differential algebraic notion of equivalence in [22] when the ground field is a field of constants. The comparison between the differential algebraic and the differential geometric settings is developed in [25]. 
In the previous definition, if endogenous transformations are replaced by Lie-Bäcklund isomorphisms, we obtain the coarser notion of orbital equivalence:

Definition 5: Two systems $(\mathcal{M}, F)$ and $(\mathcal{N}, G)$ are orbitally equivalent at $(p, q) \in \mathcal{M} \times \mathcal{N}$ if there exists a smooth mapping $\Phi$ which is a Lie-Bäcklund isomorphism from $[\mathcal{M}$, $(\operatorname{span} F)]$ to $[\mathcal{N},(\operatorname{span} G)]$ at $(p, q)$ with $q=\Phi(p)$.

$(\mathcal{M}, F)$ and $(\mathcal{N}, G)$ are orbitally equivalent if there exists a Lie-Bäcklund isomorphism $\Phi$ from $[\mathcal{D},(\operatorname{span} F)]$ to $[\mathcal{N}$, (span $G$ )], with $\mathcal{D}$ an open dense subset of $\mathcal{M}$, at every pair $(p, \Phi(p))$, for $p \in \mathcal{D}$.

Clearly, orbital equivalence includes differential equivalence. Orbital equivalence means that there exists a local one-to-one correspondence between the curves on $\mathcal{M}$ tangent to (span $F$ ) and the curves on $\mathcal{N}$ tangent to $(\operatorname{span} G)$. But, as opposed to differential equivalence, curve parameterization is not necessarily preserved.

Remark 5: Consider the case of two uncontrolled dynamics: $\dot{x}=f(x), \dot{y}=g(y)$ where $x=\left(x_{1}, \cdots, x_{n}\right), y=$ $\left(y_{1}, \cdots, y_{p}\right)$. If $n=p$, the classic straightening out theorem [1] implies that they are both locally equivalent to $\dot{\xi}_{1}=$ $0, \cdots, \dot{\xi}_{n-1}=0, \dot{\xi}_{n}=1$ and therefore equivalent in our sense.

Example 5: Consider the two classic systems $(X \times U \times$ $\left.\mathbb{R}_{m}^{\infty}, F\right)$ and $\left(Y \times V \times \mathbb{R}_{s}^{\infty}, G\right)$, respectively, describing the dynamics

$$
\begin{array}{ll}
\dot{x}=f(x, u), & (x, u) \in X \times U \subset \mathbb{R}^{n} \times \mathbb{R}^{m} \\
\dot{y}=g(y, v), & (y, v) \in Y \times V \subset \mathbb{R}^{r} \times \mathbb{R}^{s} .
\end{array}
$$

The vector fields $F, G$ are defined by

$$
\begin{gathered}
F\left(x, u, u^{(1)}, u^{(2)}, \cdots\right)=\left(f(x, u), u^{(1)}, u^{(2)}, \cdots\right) \\
G\left(y, v, v^{(1)}, v^{(2)}, \cdots\right)=\left(g(y, v), v^{(1)}, v^{(2)}, \cdots\right) .
\end{gathered}
$$

Let us recall the notations $\bar{u}=\left(u, u^{(1)}, u^{(2)}, \cdots\right)$ and $\bar{u}^{k}=$ $\left(u, u^{(1)}, \cdots, u^{(k)}\right)$, where $k$ is some finite but otherwise arbitrary integer. If the systems are equivalent the endogenous transformation $\Phi$ takes the form

$$
\begin{aligned}
\Phi(x, \bar{u}) & =\left(\varphi\left(x, \bar{u}^{k}\right), \alpha\left(x, \bar{u}^{k+1}\right), \dot{\alpha}\left(x, \bar{u}^{k+2}\right), \cdots\right) \\
& =(y, \bar{v}) .
\end{aligned}
$$

Note that since $y=\varphi\left(x, \bar{u}^{k}\right)$, and since $v$ is a solution of the implicit equation $\dot{y}-g(y, v)=0$, then $v$ may be expressed as a function of $(y, \dot{y})$ or equivalently as a function of $\left(x, \bar{u}^{k+1}\right)$. Accordingly, it is easily seen that $v^{(j)}$ is a function of $\left(x, \bar{u}^{k+j+1}\right)$ for any $j \geq 0$.

Hence $\Phi$ is completely specified by the mappings $\varphi$ and $\alpha$, i.e., by the expression of $y, v$ in terms of $x, \bar{u}$. Similarly, the inverse $\Psi$ of $\Phi$ takes the form

$$
\begin{aligned}
\Psi(y, \bar{v}) & =\left(\psi\left(y, \bar{v}^{l}\right), \beta\left(y, \bar{v}^{l+1}\right), \dot{\beta}\left(y, \bar{v}^{l+2}\right), \cdots\right) \\
& =(x, \bar{u})
\end{aligned}
$$

for some $l$.

As $\Phi$ and $\Psi$ are inverse mappings we have

$$
\begin{aligned}
\psi\left(\varphi\left(x, \bar{u}^{k}\right), \bar{\alpha}^{l}\left(x, \bar{u}^{k+l+1}\right)\right. & =x \\
\beta\left(\varphi\left(x, \bar{u}^{k}\right), \bar{\alpha}^{l+1}\left(x, \bar{u}^{k+l+2}\right)\right) & =u
\end{aligned}
$$

and vice versa. Moreover, $F$ and $G$ being $\Phi$-related implies

$$
\begin{aligned}
\dot{x} & =f\left(\psi\left(y, \bar{v}^{l}\right), \beta\left(y, \bar{v}^{l+1}\right)\right) \\
& =\frac{\partial \psi}{\partial y} g(y, v)+\sum_{i=0}^{l} \frac{\partial \psi}{\partial v^{(i)}} v^{(i+1)}
\end{aligned}
$$

for some large enough $l$.

In other words, every time $t \mapsto(x(t), u(t))$ is a trajectory of (28), then

$$
t \mapsto(y(t), v(t))=\left(\varphi\left(x(t), \bar{u}^{k}(t)\right), \alpha\left(x(t), \bar{u}^{k+1}(t)\right)\right)
$$

is a trajectory of (29), and vice versa.

The adaptation of the above interpretation to Lie-Bäcklund isomorphisms is easily done by locally expressing the time scalings as functions of a common time scale. A convenient way to adapt the above calculations consists of adding the local time as a new coordinate, namely

$$
\Phi(t, x, \bar{u})=(\tau, y, \bar{v}), \quad \Psi(\tau, y, \bar{v})=(t, x, \bar{u}) .
$$

An important property of endogenous transformations and Lie-Bäcklund isomorphisms is that they preserve the number of input channels.

Theorem 1: Consider two systems $(\mathcal{M}, F)$ and $(\mathcal{N}, G)$. If they are orbitally equivalent, then they have the same number of inputs.

Proof: Consider the Lie-Bäcklund mapping $\Phi$ from $[\mathcal{M}$, $(\operatorname{span} F)]$ to $[\mathcal{N}$, (span $G)]$. It can be expressed in local coordinates as

$$
\begin{aligned}
\Phi(t, x, \bar{u})= & (\tau, y, \bar{v}) \\
= & \left(\tau\left(t, x, \bar{u}^{k}\right), \varphi\left(t, x, \bar{u}^{k}\right), \alpha\left(t, x, \bar{u}^{k+1}\right),\right. \\
& \left.\dot{\alpha}\left(t, x, \bar{u}^{k+2}\right), \cdots\right)
\end{aligned}
$$

where $k$ is just a fixed "large enough" integer. Denoting by

$$
\begin{aligned}
& \Phi_{\mu}\left(t, x, \bar{u}^{k+\mu+1}\right) \\
& \quad=\left(\tau\left(t, x, \bar{u}^{k}\right), \varphi\left(t, x, \bar{u}^{k}\right), \bar{\alpha}^{\mu}\left(t, x, \bar{u}^{k+\mu+1}\right)\right)
\end{aligned}
$$

for $\mu \geq 0$, we have

$$
\left(\tau, y, \bar{v}^{\mu}\right)=\Phi_{\mu}\left(t, x, \bar{u}^{k+\mu+1}\right) .
$$

Because $\Phi$ is invertible, $\Phi_{\mu}$ is a surjection for all $\mu$. Hence the dimension of the source is greater than the dimension of the target

$$
\forall \mu, \quad 1+n+m(k+\mu+2) \geq 1+n^{\prime}+s(\mu+1)
$$

where $n^{\prime}$ is the dimension of $Y$, or

$(m-s) \mu+(1+n+m(k+2))-\left(1+n^{\prime}+s\right) \geq 0, \quad \forall \mu \geq 0$.

Clearly, it implies that $m \geq s$ since otherwise $(m-s) \mu$ could be made arbitrarily negative for $\mu$ large enough. Using the same idea on $\Psi$ leads to $s \geq m$.

For controllable linear systems, we have the following more precise result.

Theorem 2: Two (classic) linear controllable systems are differentially equivalent if, and only if, they have the same number of inputs. 
Proof: The necessity follows from Theorem 1. Conversely, since a linear controllable system admits a Brunovský controllability canonical form (see, e.g., [41]), it suffices to prove that two such canonical forms with $m$ inputs are differentially equivalent. Consider two such forms: $x_{i}^{\left(\mu_{i}\right)}=u_{i}$ and $y_{i}^{\left(\nu_{i}\right)}=v_{i}, i=1, \cdots, m$ where the $\mu_{i}$ 's and $\nu_{i}$ 's are the associated controllability indexes. It is then straightforward to remark that they are both differentially equivalent to the trivial system $\left(\mathbb{R}_{m}^{\infty}, F_{m}\right)$ (see Example 1 ).

\section{Flatness}

Recall from Example 1 that a trivial system is a system $\left(\mathbb{R}_{s}^{\infty}, F_{s}\right)$, where $F_{s}\left(y, y^{(1)}, y^{(2)}, \cdots\right)=\left(y^{(1)}, y^{(2)}, y^{(3)}\right.$, $\cdots)$, with $y \in \mathbb{R}^{s}$.

Definition 6: The control system $(\mathcal{M}, F)$ is said to be differentially flat (or shortly flat) around $p$, if, and only if, it is equivalent to a trivial system in a neighborhood of $p$. It is called differentially flat if it is differentially flat around every $p$ of an open dense subset of $\mathcal{M}$.

The set $y=\left\{y_{j} \mid j=1, \cdots, s\right\}$ is called a flat or linearizing output of $\mathcal{M}$.

Definition 7: The control system $(\mathcal{M}, F)$ is said to be orbitally flat around a point $p$ if, and only if, it is orbitally equivalent around $p$ to a trivial system. Orbital flatness means orbital flatness around every point of an open dense subset of $\mathcal{M}$

We immediately deduce from Theorems 1 and 2 the following result.

Corollary 3: Consider a flat system (orbitally or differentially). The number of components of a flat output is equal to the number of input channels.

Reformulating the Theorem 2, yields the following.

Corollary 4: A (classic) linear system is flat if, and only if, it is controllable.

Proof: Consider a flat classic linear system $\dot{x}=A x+B u$, the notations being those of the Example 2, and, for the trivial system, of the Example 1. If the system is not controllable, there exists a linear differential equation

$$
E_{0} x+E_{1} \dot{x}+\cdots+E_{\alpha} x^{(\alpha)}=0
$$

independent of the input $u$, with $\alpha \geq 0$ and at least one of the matrices $E_{j}, 0 \leq j \leq \alpha$, nonzero. According to the equivalence to a trivial system, we must have $x=\Phi\left(\bar{y}^{\beta}\right)$, for some integer $\beta \geq 0$, with, as before, $\bar{y}^{\beta}=\left(y, \dot{y}, \cdots, y^{(\beta)}\right)$ and $\Phi$ a submersion. Easy calculations show that, for every integer $k, x^{(k)}=A_{k}\left(\bar{y}^{\beta+k-1}\right)+\left(\partial \Phi / \partial \bar{y}^{\beta}\right)\left(d^{k} \bar{y}^{\beta} / d t^{k}\right)$ where $A_{k}$ is an expression involving at most derivatives of $y$ up to the order $\beta+k-1$. Replacing in (31), we get $E_{0} \Phi\left(\bar{y}^{\beta}\right)+\cdots+$ $E_{\alpha}\left(A_{k}\left(\bar{y}^{\beta+k-1}\right)+\left(\partial \Phi / \partial \bar{y}^{\beta}\right)\left(d^{k} \bar{y}^{\beta} / d t^{k}\right)\right)=0$. According to the independence of the components of $\bar{y}^{\alpha+\beta}$ and to the fact that the mapping $\Phi$ is a submersion, $\partial \Phi / \partial \bar{y}^{\beta}$ is a full rank matrix in an open dense subset and thus we must have $E_{\alpha}=0$. Following the same lines, we get $E_{\alpha}=E_{\alpha-1}=\cdots=E_{1}=$ 0 . Finally, we also have $E_{0} \Phi\left(\bar{y}^{\beta}\right)=0$, again by the fact that $\Phi$ is a submersion, since otherwise, the components of $\bar{y}^{\beta}$ would be dependent by the implicit function theorem, which achieves to prove the controllability. According to Theorem 2, the proof is complete.

The controllability of differentially flat systems is now related to the well-known strong accessibility property of nonlinear systems due to Sussmann and Jurdjevic [74] (see, also, [32], [36], [58], and, for a presentation of the strong accessibility property in the language of infinite jets and prolongations, see [17] and [26]).

Corollary 5: If a classic nonlinear system is differentially flat around $p$, then it satisfies the strong accessibility property at $p$.

Proof: Our system is differentially equivalent around $p$ to a trivial system via an endogenous transformation $\Phi ; \Phi$ and its inverse $\Psi$ give a continuous local isomorphism. Therefore, the image by $\Psi$ of an open neighborhood of $\Phi(p)$ is an open neighborhood of $p$, which means that every trajectory of the trivial system starting from $\Phi(p)$ and passing through any point $q$ of this neighborhood is the image of a trajectory of the nonlinear system joining the point $p$ and $\Psi(q)$. Therefore, the interior of the reachable set from $p$ is an open neighborhood of $p$ and, as a consequence of [36] and [58], the strong accessibility condition holds true. ${ }^{3}$

Example 6: Consider the control system

$$
\dot{x}_{1}=u, \quad \dot{x}_{2}=(u)^{2} .
$$

Proposition 6: System (32) is orbitally flat but not differentially flat.

Proof: Consider the mapping

$$
\begin{aligned}
& \Phi: \mathbb{R} \times \mathbb{R} \times \mathbb{R}^{\infty} \quad \longrightarrow \mathbb{R} \times \mathbb{R}^{\infty} \\
& \left(x_{1}, x_{2},\left(u^{(\mu)}\right)_{\mu \geq 0}\right) \mapsto\left(\tau,\left(\frac{d^{(\mu)} y}{d \tau^{(\mu)}}\right)_{\mu \geq 0}\right)
\end{aligned}
$$

with $\tau=x_{1}$ and $y=x_{2}$.

Clearly, $\Phi$ is a Lie-Bäcklund isomorphism since $d t / d \tau=$ $1 / u, y^{(1)}=d y / d \tau=d x_{2} / d x_{1}=u$ and the derivatives of $y$ with respect to $\tau$ are computed by the formula $y^{(k)}=$ $(d / d t) y^{(k-1)}(d t / d \tau)=(1 / u)(d / d t) y^{(k-1)}$ for every $k \geq 1$.

Conversely, $x_{1}=\tau, x_{2}=y, u=y^{(1)}$, and $t$ is recovered by $d t / d \tau=1 / y^{(1)}$. Therefore, (32) is orbitally flat. On the other hand, it cannot be differentially flat since, for single input systems, it implies static feedback linearizability [10], which is clearly not the case (see [34] and [40]), and the assertion is proved.

An example of a nonorbitally flat system can be found in Hilbert in a different context [33]. Comparable ideas have also been exploited in [22], [66], and [72] to derive the ruledmanifold criterion, which is a most useful necessary condition for differential flatness.

Example 7-VTOL Aircraft (Continued): As shown in [49], this time-invariant system is differentially flat. The flat output is the Huygens oscillation center

$$
\left(y_{1}, y_{2}\right)=(x+\varepsilon \sin \theta, z+\varepsilon \cos \theta) .
$$

\footnotetext{
${ }^{3}$ Our proof demonstrates that the controllability of a differentially flat system is stronger than the strong accessibility property; as a matter of fact, we can reach the points of an open subset around the corresponding point in the state space.
} 
Consider the manifold $\mathcal{M}$ with coordinates $\left(x, v_{x}, z, v_{z}, \theta\right.$, $\left.v_{\theta},\left(u_{1}^{(\nu)}, u_{2}^{(\nu)}\right)_{\nu \geq 0}\right)$ and vector field $F$ defined by (26). Let us also consider the trivial system $\left(\mathbb{R}_{2}^{\infty}, F_{2}\right)$ with $F_{2}$ defined as in Example 1.

The mapping

$$
\begin{aligned}
\Phi: & \left(x, v_{x}, z, v_{z}, \theta, v_{\theta},\left(u_{1}^{(\nu)}, u_{2}^{(\nu)}\right)_{\nu \geq 0}\right) \\
& \mapsto\left(y_{1}^{(\mu)}, y_{2}^{(\mu)}\right)_{\mu \geq 0}
\end{aligned}
$$

where we have used the convention $y_{i}^{0}=y_{i}, i=1, \cdots, m$, and

$$
\begin{aligned}
y_{1} & =x+\varepsilon \sin \theta \\
y_{2} & =z+\varepsilon \cos \theta \\
y_{1}^{(1)} & =v_{x}+\varepsilon v_{\theta} \cos \theta \\
y_{2}^{(1)} & =v_{z}-\varepsilon v_{\theta} \sin \theta \\
y_{1}^{(2)} & =\left(u_{1}-\varepsilon\left(v_{\theta}\right)^{2}\right) \sin \theta \\
y_{2}^{(2)} & =-1+\left(u_{1}-\varepsilon\left(v_{\theta}\right)^{2}\right) \cos \theta \\
y_{1}^{(3)} & \left.=\frac{d}{d t}\left(\left(u_{1}-\varepsilon\left(v_{\theta}\right)^{2}\right) \sin \theta\right)\right) \\
y_{2}^{(3)} & =\frac{d}{d t}\left(\left(u_{1}-\varepsilon\left(v_{\theta}\right)^{2}\right) \cos \theta\right) \\
y_{1}^{(4)} & =\frac{d^{2}}{d t^{2}}\left(\left(u_{1}-\varepsilon\left(v_{\theta}\right)^{2}\right) \sin \theta\right) \\
y_{2}^{(4)} & =\frac{d^{2}}{d t^{2}}\left(\left(u_{1}-\varepsilon\left(v_{\theta}\right)^{2}\right) \cos \theta\right)
\end{aligned}
$$

is an isomorphic endogenous transformation such that $F$ and $F_{2}$ are $\Phi$-related. The inverse mapping is based on the relations (13), which, in our notation, reads

$$
\begin{aligned}
& x=y_{1}-\varepsilon \frac{y_{1}^{(2)}}{\sqrt{\left(y_{1}^{(2)}\right)^{2}+\left(y_{2}^{(2)}+1\right)^{2}}} \\
& z=y_{2}-\varepsilon \frac{y_{2}^{(2)}+1}{\sqrt{\left(y_{1}^{(2)}\right)^{2}+\left(y_{2}^{(2)}+1\right)^{2}}} \\
& \theta=\arctan \left(\frac{y_{1}^{(2)}}{y_{2}^{(2)}+1}\right) .
\end{aligned}
$$

As noted in [22], differential flatness means that the state and input may be completely recovered from the flat output without integrating the system differential equations. The consequences on the solution to the motion planning problem as well as for trajectory stabilization are immediately understood. The reader may refer once more to [22], [50], and the bibliography therein for an extensive collection of examples illustrating the various applications of flatness.

As a consequence of the flatness of the VTOL and of the equivalence relation described in Section I, we have proved the following.
Proposition 7: Systems (1), (2), (6), (9), and (10) are all differentially flat.

\section{INTERPRETATION OF EQUIVALENCE IN TERMS OF FEEDBACK}

Consider the two systems $\left(X \times U \times \mathbb{R}_{m}^{\infty}, F\right)$ and $(Y \times V \times$ $\left.\mathbb{R}_{s}^{\infty}, G\right)$, respectively, describing the dynamics

$$
\begin{array}{ll}
\dot{x}=f(x, u), & (x, u) \in X \times U \subset \mathbb{R}^{n} \times \mathbb{R}^{m} \\
\dot{y}=g(y, v), & (y, v) \in Y \times V \subset \mathbb{R}^{r} \times \mathbb{R}^{s} .
\end{array}
$$

The vector fields $F, G$ are defined by

$$
\begin{gathered}
F\left(x, u, u^{(1)}, u^{(2)}, \cdots\right)=\left(f(x, u), u^{(1)}, u^{(2)}, \cdots\right) \\
G\left(y, v, v^{(1)}, v^{(2)}, \cdots\right)=\left(g(y, v), v^{(1)}, v^{(2)}, \cdots\right) .
\end{gathered}
$$

Note that the general case can be reduced to the above case as follows. We have seen that a system may be locally described by a manifold $X \times \mathbb{R}_{m}^{\infty}$ with coordinates $(\zeta, \bar{u})$ and a vector field of the form (25). Since $g$ contains only a finite number of derivatives of $u$, let us denote by $r$ the highest order of derivation and $v=u^{(r)}$. Then setting $x=\left(\zeta, u, \dot{u}, \cdots, u^{(r-1)}\right)$, we easily see that the vector field in these new coordinates is expressed as a classic vector field. We thus only sketch the results in the classic case since they easily extend to the general case by the same remark. In order to avoid some technicalities related to the intrinsic definition of a general dynamic feedback, ${ }^{4}$ the next result, though valid for general systems, is only stated in the classic setting.

If systems (36) and (37) are (differentially) equivalent, we are going to show that it is possible to go from (36) to (37) and vice versa by a dynamic feedback

$$
\begin{aligned}
& u=\sigma(x, z, w) \\
& \dot{z}=a(x, z, w)
\end{aligned}
$$

with $z \in Z \subset \mathbb{R}^{q}$, in the usual sense, namely by a diffeomorphism of the extended state space $X \times Z$.

We say that the dynamic feedback (38) is endogenous if the original system (36) is differentially equivalent to the closedloop system (36)-(38). Such a feedback is called endogenous because the new $z$ variables it contains can be expressed as functions of the state and (finitely many) derivatives of the input.

Of course we cannot hope to go from one dynamics to the other without changing the state dimension. But this is in some sense the only thing we lose.

Theorem 8: Assume that the systems $\left(X \times U \times \mathbb{R}_{m}^{\infty}, F\right)$ and $\left(Y \times V \times \mathbb{R}_{s}^{\infty}, G\right)$ are differentially equivalent. Then, $s=m$ and there exists an endogenous dynamic feedback such that the closed-loop system (36)-(38) is diffeomorphic to (37) prolonged by sufficiently many integrators.

\footnotetext{
${ }^{4}$ It can be shown (see [20] and [21]) that a general dynamic feedback is a Lie-Bäcklund correspondence (see [80]).
} 
Here "(37) prolonged by sufficiently many integrators" means

$$
\begin{gathered}
\dot{y}=g(y, v) \\
\dot{v}=v^{(1)} \\
\dot{v}^{(1)}=v^{(2)} \\
\vdots \\
\dot{v}^{(\mu)}=w
\end{gathered}
$$

for $\mu$ large enough.

Proof: Remember that it suffices to prove the result in the classic case. The proof follows [45] and [46]. Let $\tilde{y}=\left(y, \bar{v}^{\mu}\right)=\left(y, v, v^{(1)}, \cdots, v^{(\mu)}\right)$ and $w=v^{(\mu+1)}$. Using the notations of Example 5, we see that, for $\mu$ large enough, $\psi$ depends only on $\tilde{y}$ and $\beta$ only on $(\tilde{y}, w)$, i.e., the endogenous transformation $\Psi$ takes the form

$$
\Psi\left(\tilde{y}, w, w^{(1)}, \cdots\right)=\left(\psi(\tilde{y}), \beta(\tilde{y}, w), \dot{\beta}\left(\tilde{y}, \bar{w}^{1}\right), \cdots\right)
$$

and (30) now reads

$$
f(\psi(\tilde{y}), \beta(\tilde{y}, w))=\frac{\partial \psi}{\partial(\tilde{y})} \tilde{g}(\tilde{y}, w)
$$

where $\tilde{g}=\left(g, \bar{v}^{\mu}\right)$ is the prolonged vector field corresponding to (39).

Let $\tilde{y}=\left(\tilde{y}_{a}, \tilde{y}_{b}\right)$ be a splitting of the components of $\tilde{y}$ such that the mapping

$$
\tilde{y} \mapsto K(\tilde{y})=\left(\psi(\tilde{y}), \tilde{y}_{b}\right)
$$

is invertible (such a splitting exists because $\varphi$, being a block of components of the invertible mapping $\Phi$, is full rank). Apply now the dynamic feedback

$$
\begin{aligned}
& u=\beta\left(K^{-1}(x, z), w\right) \\
& \dot{z}=\tilde{g}_{b}\left(K^{-1}(x, z), w\right)
\end{aligned}
$$

where $\tilde{g}_{b}$ stands for the projection of $\tilde{g}$ corresponding to $\tilde{y}_{b}$, to get the closed-loop dynamics

$$
\left(\begin{array}{c}
\dot{x} \\
\dot{z}
\end{array}\right)=\tilde{f}(x, z, w)=\left(\begin{array}{c}
f\left(x, \beta\left(K^{-1}(x, z), w\right)\right) \\
\tilde{g}_{b}\left(K^{-1}(x, z), w\right)
\end{array}\right) .
$$

Using (40), we have

$$
\begin{aligned}
\tilde{f}(K(\tilde{y}), w) & =\left(\begin{array}{c}
f(\psi(\tilde{y}), \beta(\tilde{y}, w)) \\
\tilde{g}_{b}(\tilde{y}, w)
\end{array}\right) \\
& =\left(\begin{array}{cc}
\frac{\partial \psi}{\partial \tilde{y}}(\tilde{y}) & 0 \\
0 & I
\end{array}\right) \tilde{g}(\tilde{y}, w) \\
& =\frac{\partial K}{\partial \tilde{y}}(\tilde{y}) \tilde{g}(\tilde{y}, w)
\end{aligned}
$$

which means that (39) and (41) are diffeomorphic.

Finally, since $y=\varphi\left(x, \bar{u}^{\nu}\right)$ and $v=\alpha\left(x, \bar{u}^{\nu+1}\right)$ for some $\nu$, we get that $z=\tilde{y}_{b}=\tilde{\varphi}_{b}\left(x, \bar{u}^{\nu+\mu+1}\right)$, where $\tilde{\varphi}$ stands for the mapping $\left(\varphi, \alpha, \cdots, \alpha^{(\nu)}\right)$ and where $\tilde{\varphi}_{b}$ is the projection of $\tilde{\varphi}$ corresponding to $\tilde{y}_{b}$. It proves that (36) and its closedloop dynamics (41) are differentially equivalent and thus that the corresponding dynamic feedback is endogenous.
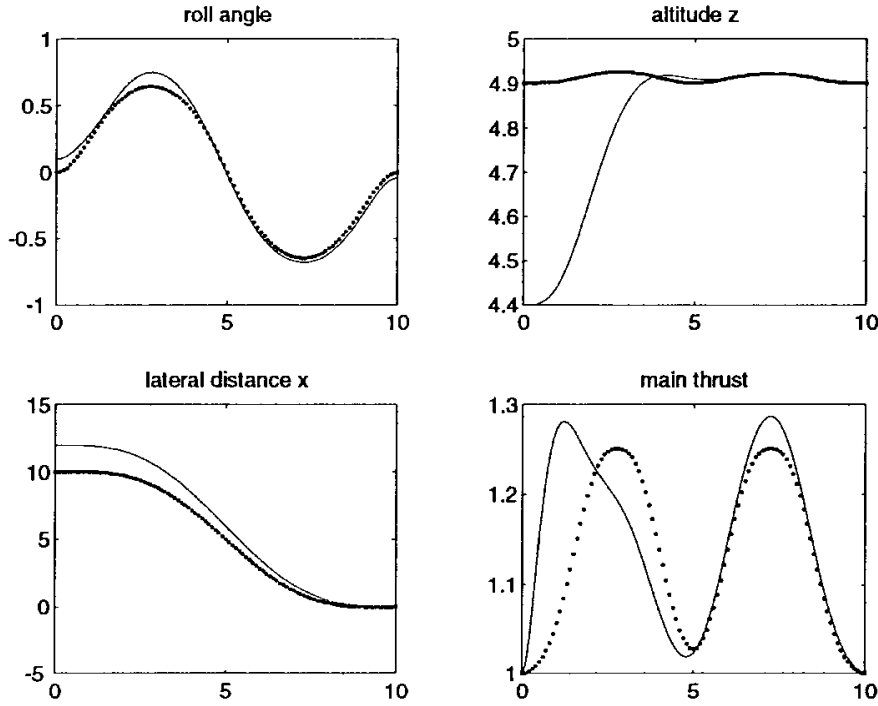

Fig. 3. The reference trajectories (dashed) and the closed-loop trajectories (continuous) for $\theta, x, z$, and the main thrust $u_{1}$.

Remark 6: The previous theorem may easily be extended to orbital equivalence. The proof is left to the reader.

Since any prolongation of a controllable linear system is again a controllable linear system (see [70]), we are lead to Corollary 9.

Corollary 9: If a system is differentially flat, there exists an endogenous dynamic feedback such that the closed-loop system is diffeomorphic to a linear controllable system.

This result slightly clarifies previous works in dynamic feedback linearization due to Charlet et al. [10], [11].

Example 8-VTOL Aircraft (End): According to (35), the state of (1), i.e., $\left(x, v_{x}, z, v_{z}, \theta, v_{\theta}\right)$ is a function of the linearizing output $\left(y_{1}, y_{2}\right)$ and its derivatives up to order $\alpha=3$. Thus, according to the above method, there exists an endogenous dynamic feedback leading to the following closed-loop system $(\alpha+1=4)$ :

$$
y_{1}^{(4)}=v_{1}, \quad y_{2}^{(4)}=v_{2} .
$$

It yields the following linearizing dynamic feedback $(q=2)$ : let us denote $\eta_{1}=u_{1}-\varepsilon\left(v_{\theta}\right)^{2}$ and $\dot{\eta}_{1}=\eta_{2}$. By (34), we have

$$
\begin{aligned}
& y_{1}^{(2)}=\eta_{1} \sin \theta \\
& y_{1}^{(3)}=\eta_{2} \sin \theta+\eta_{1} v_{\theta} \cos \theta \\
& y_{2}^{(2)}=-1+\eta_{1} \cos \theta \\
& y_{2}^{(3)}=\eta_{2} \cos \theta-\eta_{1} v_{\theta} \sin \theta
\end{aligned}
$$

and thus

$$
\begin{aligned}
y_{1}^{(4)} & =\dot{\eta}_{2} \sin \theta+2 \eta_{2} v_{\theta} \cos \theta+\eta_{1} u_{2} \cos \theta-\eta_{1} v_{\theta}^{2} \sin \theta \\
& =v_{1} \\
y_{2}^{(4)} & =\dot{\eta}_{2} \cos \theta-2 \eta_{2} v_{\theta} \sin \theta-\eta_{1} u_{2} \sin \theta-\eta_{1} v_{\theta}^{2} \cos \theta \\
& =v_{2} .
\end{aligned}
$$

Inverting this linear system with respect to $\dot{\eta}_{2}$ and $u_{2}$ yields 


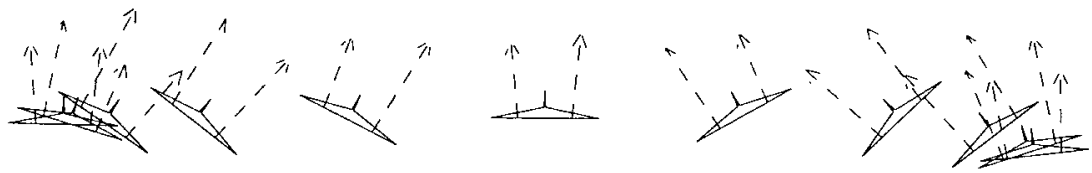

Fig. 4. An animation of the dynamic behavior of the VTOL aircraft, with the intensities of the thrust of each reactor and of the resultant applied to the center of mass.

the following dynamic compensator:

$$
\begin{aligned}
& \dot{\eta}_{1}=\eta_{2} \\
& \dot{\eta}_{2}=v_{1} \sin \theta+v_{2} \cos \theta+\left(v_{\theta}\right)^{2} \eta_{1} \\
& u_{1}=\eta_{1}+\varepsilon\left(v_{\theta}\right)^{2} \\
& u_{2}=\frac{1}{\eta_{1}}\left(v_{1} \cos \theta-v_{2} \sin \theta-2 v_{\theta} \eta_{2}\right) .
\end{aligned}
$$

Notice that this dynamic feedback is not a simple prolongation where time derivatives of the control $\left(u_{1}, u_{2}\right)$ are added to the state. For this system, classic results show that, for any $\mu_{1} \geq 0$ and $\mu_{2} \geq 0$, the prolonged system (1) with $u_{1}^{\left(\mu_{1}\right)}=v_{1}$ and $u_{2}^{\left(\mu_{1}\right)}=v_{2}$, is not linearizable via static feedback. This point constitutes one of the major difficulties for finite characterization of flat systems.

Let us now show how the dynamic compensator (42) may be used to track given reference trajectories.

Set $X=\left(x, v_{x}, z, v_{z}, \theta, v_{\theta}\right)$, the state of the VTOL model. Assume that the goal is simply to go from an initial state $X_{i}$ at time $t_{i}$ to a final state $X_{f}$ at time $t_{f}$. Thanks to the flatness of the system, the state and input can be expressed in the coordinates $\left(y, \dot{y}, \ddot{y}, y^{(3)}, y^{(4)}\right)$. Therefore, the constraints on the state at times $t_{i}$ and $t_{f}$ can be translated into constraints on $y$ and its derivatives up to fourth order. For example, if we want to go from a given rest point to another rest point, any curve satisfying these constraints, for instance a polynomial of degree seven with respect to time, namely $y_{j}(t)=\sum_{k=0}^{7} a_{j, k} t^{k}, j=1,2$, satisfying $y_{1}\left(t_{i}\right)=x_{i}+\varepsilon \sin \theta_{i}, y_{2}\left(t_{i}\right)=z_{i}+\varepsilon \cos \theta_{i}, y_{j}^{(1)}\left(t_{i}\right)=$ $y_{j}^{(2)}\left(t_{i}\right)=y_{j}^{(3)}\left(t_{i}\right)=0, j=1,2$, and $y_{1}\left(t_{f}\right)=x_{f}+\varepsilon \sin \theta_{f}$, $y_{2}\left(t_{f}\right)=z_{f}+\varepsilon \cos \theta_{f}, y_{j}^{(1)}\left(t_{f}\right)=y_{j}^{(2)}\left(t_{f}\right)=y_{j}^{(3)}\left(t_{f}\right)=0$, $j=1,2$, will then generate a suitable trajectory of the system by solving two systems of linear equations for the $2 \times 8$ coefficients $\left\{a_{j, 0}, \cdots, a_{j, 7}, j=1,2\right\}$ (see [22] for more details). Let us denote by $y^{*}=\left(y_{1}^{*}, y_{2}^{*}\right)$ the corresponding reference trajectory. Here, we want such a transfer to take place in $10 \mathrm{~s}$ (remember that the distances are normalized with respect to the gravitation field, hence a length of 10 represents about $100 \mathrm{~m}$ ).

At a second step, we close the loop by stabilizing the reference trajectory $y^{*}$. It suffices to use (42) with

$$
v_{i}=\left(y_{i}^{*}\right)^{(4)}+\sum_{j=0}^{3} k_{i, j}\left(y_{i}^{(j)}-\left(y_{i}^{*}\right)^{(j)}\right), \quad i=1,2,
$$

the coefficients $k_{i, j}$ being chosen such that the linear time invariant error dynamics

$$
e_{i}^{(4)}=\sum_{j=0}^{3} k_{i, j} e_{i}^{(j)}, \quad i=1,2
$$

with $e_{i}^{(j)}=y_{i}^{(j)}-\left(y_{i}^{*}\right)^{(j)}$, are stable.
We illustrate this approach with a simple simulation: the initial rest point is $x_{i}=10, z_{i}=5$ and the final one is $x_{f}=0, z_{f}=0$ with $t_{f}-t_{i}=10 \mathrm{~s}$. To show that perturbations may not alter the performances of the closed-loop system, the system is given erroneous initial conditions. The simulation results giving $(\theta, x, z)$ and the thrust are displayed in Fig. 3 and an animation is presented in Fig. 4.

Remark 7: The flat dynamics (16) with output $y=$ $\left(y_{1}, \cdots, y_{m}\right)$ is a square left and right input-output invertible system (see, e.g., [15] and [58]), where, moreover, any component of $u$ or $x$ may be, by definition, recovered from $y$ without integrating any differential equation: we will say that it possesses a trivial zero-dynamics or a trivial residual dynamics (see [36] and [58] for a general definition of zero-dynamics) since we are, for instance, not necessarily working at an equilibrium point. It yields the following.

Proposition 10: The system (16) is differentially flat around a point if, and only if, it is possible to find an $m$-dimensional output $y$, which smoothly depends around that point on $x, u$, and a finite number of derivatives of $u$, such that the resulting square system is left and right input-output invertible, with a trivial zero-dynamics.

This proposition, which is an easy adaptation of a result of [37], may be considered as an alternative definition of a flat system. However, with such a definition, the underlying feedback equivalence relation as well as the geometry of the problem would be less apparent.

\section{CONCLUSION}

We have proposed a differential geometric approach for investigating a new system equivalence, the Lie-Bäcklund equivalence, which can be realized by endogenous feedback, a special type of dynamic feedback. Such a Lie-Bäcklund equivalence is shown to be useful to reduce the dimension of a complex system and to study differentially or orbitally flat systems.

Flat systems should be regarded as justified by the wealth of realistic case studies it is capable of handling, as already mentioned in the introduction. Unfortunately, up to now, no general checkable conditions for flatness have been obtained. This is perhaps the main mathematical problem in this setting and constitutes a currently active field of research, as can be seen in [4], [11], [37], [39], [50]-[52], [56], [57], [61]-[63], [69], and [72].

More generally, the classification of nonlinear systems, including nonflat systems, via Lie-Bäcklund equivalence remains to be done. 


\section{REFERENCES}

[1] R. Abraham, J. E. Marsden, and T. Ratiu, Manifolds, Tensor Analysis, and Applications, 2nd ed. New York: Springer, 1988.

[2] R. L. Anderson and N. H. Ibragimov, Lie-Bäcklund Transformations in Applications. Philadelphia, PA: SIAM, 1979.

[3] B. d'Andrea-Novel, P. Martin, and R. Sépulchre, "Full linearization of a class of mechanical systems via dynamic state feedback," in Recent Advances in Mathematical Theory of Systems, Control, Networks and Signal Processing II-Proc. Int. Symp. MTNS'91. Tokyo, Japan: Mita, 1992.

[4] E. Aranda-Bricaire, C. H. Moog, and J.-B. Pomet, "A linear algebraic framework for dynamic feedback linearization," IEEE Trans. Automat. Contr., vol. 40, pp. 127-132, 1995.

[5] R. Baron, J. Lévine, and M. Mastail, "Modeling and control of a fish extrusion process," in Proc. 1st IMACS/IFAC Conf. Mathematical Modeling and Simulation in Agriculture and Bio-Industries, Bruxelles, Belgium, May 1995.

[6] L. Bitauld, M. Fliess, and J. Lévine, "A flatness based control synthesis of linear systems and application to windshield wipers," in Proc. ECC, Brussels, Belgium, July 1997.

[7] S. L. Campbell, "High order index differential algebraic equations," $J$. Mechanics Structures Machines, vol. 23, pp. 199-222, 1995.

[8] E. Cartan, "Sur l'équivalence absolue de certains systèmes d'équations différentielles et sur certaines familles de coubres," Bull. Soc. Math. France, vol. 42, pp. 12-48, 1914; also in Oeuvres Complètes, CNRS, Paris, 1984, pt. II, vol. 2, pp. 1133-1168.

[9] __ "Les problèmes d'équivalence," Selecta, Séminaire de Math., exposé D, pp. 113-136, 1937; also in Oeuvres Complètes, CNRS, Paris, 1984, pt. II, vol. 2, pp. 1311-1334.

[10] B. Charlet, J. Lévine, and R. Marino, "On dynamic feedback linearization," Syst. Contr. Lett., vol. 13, pp. 143-151, 1989.

[11] "Sufficient conditions for dynamic state feedback linearization," SIAM J. Contr. and Optim., vol. 29, no. 1, pp. 38-57, 1991.

[12] D. Cheng, "Linearization with dynamic compensation," J. Syst. Sci., vol. 7, pp. 63-83, 1987

[13] J. Dieudonné, Foundations of Modern Analysis. New York: Academic, 1960.

[14] M. Fliess, "Generalized controller canonical forms for linear and nonlinear dynamics," IEEE Trans. Automat. Contr., vol. 35, pp. 994-1001, 1990.

[15] M. Fliess and S. T. Glad, "An algebraic approach to linear and nonlinear control," in Essays on Control: Perspectives in the Theory and its Applications, H. J. Trentelman and J. C. Willems, Eds. Boston, MA: Birkhäuser, 1993, pp. 223-267.

[16] M. Fliess, J. Lévine, Ph. Martin, F. Ollivier, and P. Rouchon, "Controlling nonlinear systems by flatness," in Systems and Control in the Twenty-First Century, C. I. Byrnes, B. N. Datta, D. S. Gilliam, and C. F. Martin, Eds. Boston, MA: Birkhäuser, 1997, pp. 137-154.

[17] , "A remark on nonlinear accessibility conditions and infinite prolongations," Syst. Contr. Lett., vol. 31, pp. 77-83, 1997.

[18] M. Fliess, J. Lévine, Ph. Martin, and P. Rouchon, "Sur les systèmes non linéaires différentiellement plats," C.R. Acad. Sci. Paris, vol. I-315, pp. 619-624, 1992

[19] _ "On differentially flat nonlinear systems," in Proc. IFAC-Symp. NOLCOS'92, Bordeaux, France, pp. 408-412.

[20] _ , "Linéarization par bouclage dynamique et transformations de Lie-Bäcklund," C.R. Acad. Sci. Paris, vol. I-317, pp. 981-986, 1993.

[21] _ _ "Nonlinear control and Lie-Bäcklund transformations: Toward a new differential geometric standpoint," in Proc. 33th IEEE Conf. Decision Control, Lake Buena Vista, FL, 1994, pp. 339-344.

[22] _ _ "Flatness and defect of nonlinear systems: Introductory theory and examples," Int. J. Contr., vol. 61, no. 6, pp. 1327-1361, 1995.

[23] — "Design of trajectory stabilizing feedback for driftless flat systems," in Proc. 3rd European Control Conf., Rome, Italy, 1995, pp. $1882-1887$.

[24] _ "A Lie-Bäcklund approach to dynamic feedback equivalence and flatness," in Robust Control via Variable Structure and Lyapunov Techniques, Lect. Notes in Control Information Sci., vol. 217, F. Garofallo and L. Glielmo, Eds. London, U.K.: Springer, 1996, pp. $245-258$.

[25] _ _ "Deux applications de la géométrie locale des diffiétés," Annales Inst. Henri Poincaré, Phys. Théor., vol. 66, no. 3, pp. 275-292, 1997.

[26] _ "Nonlinear control and diffieties with an application to physics," in Secondary Calculus and Cohomological Physics, Contemporary Mathematics no. 219, M. Henneaux, J. Krasil'schchik, A Vinogradov, Eds. American Math. Soc., 1998, pp. 81-92.
[27] M. Fliess, J. Lévine, and P. Rouchon, “A generalized state variable representation for a simplified crane description," Int. J. Contr., vol. 58 , pp. 277-283, 1993.

[28] M. Fliess, and J. Rudolph, "Corps de Hardy et observateurs asymptotiques locaux pour systèmes différentiellement plats," C.R. Acad. Sci. Paris, vol. II-324, pp. 513-519, 1997.

[29] R. Gardner and W. Shadwick, "The GS algorithm for exact linearization to Brunovsky normal form," IEEE Trans. Automat. Contr., vol. 37, pp. 224-230, 1992.

[30] M. Golubtisky and V. Guillemin, Stable Mappings and Their Singularties. New York: Springer, 1973.

[31] J. Hauser, S. Sastry, and G. Meyer, "Nonlinear control design for slightly nonminimum phase systems: Application to V/STOL aircraft," Automatica, vol. 28, pp. 665-679, 1992.

[32] R. Hermann and A. J. Krener, "Nonlinear controllability and observability," IEEE Trans. Automat. Contr., vol. 22, pp. 728-740, 1977.

[33] D. Hilbert, "Über den Begriff der Klasse von Differentialgleichungen," Math. Ann., vol. 73, pp. 95-108, 1912; also in Gesammelte Abhandlungen, Chelsea, NY, vol. III, pp. 81-93, 1965.

[34] L. R. Hunt, R. Su, and G. Meyer, "Design for multi-input nonlinear systems," in Differential Geometric Control Theory, R. Brockett, R. Millman, and H. J. Sussmann, Eds. Basel: Birkhäuser, 1983, pp. 268-298.

[35] N. H. Ibragimov, Transformation Groups Applied to Mathematical Physics. Boston, MA: Reidel, 1985.

[36] A. Isidori, Nonlinear Control Systems, 3rd ed. New York: Springer, 1995.

[37] A. Isidori, C. Moog, and A. De Luca, "A sufficient condition for full linearization via dynamic state feedback," in Proc. 25th IEEE Conf. Decision Control, Athens, Greece, 1986, pp. 203-207.

[38] B. Jakubczyk, "Remarks on equivalence and linearization of nonlinear systems," in Proc. IFAC-Symp. NOLCOS'92, Bordeaux, France, pp. 393-397.

[39] _ , "Invariants of dynamic feedback and free systems," in Proc. 2nd European Control Conf. ECC'93, Groningen, The Netherlands, pp. $1510-1513$

[40] B. Jakubczyk and W. Respondek, "On linearization of control systems," Bull. Acad. Pol. Sci., Ser. Sci. Math., vol. 18, pp. 517-522, 1980.

[41] T. Kailath, Linear Systems. Englewood Cliffs, NJ: Prentice-Hall, 1980.

[42] I. S. Krasil'shchik, V. V. Lychagin, and A. M. Vinogradov, Geometry of Jet Spaces and Nonlinear Partial Differential Equations. New York: Gordon and Breach, 1986.

[43] J. Lévine, J. Lottin, and J. C. Ponsart, "A nonlinear approach to the control of magnetic bearings," IEEE Trans. Contr. Syst. Technol., Special Issue on Magnetic Bearing Control, vol. 4, pp. 524-544, 1996.

[44] J. Lévine, P. Rouchon, G. Yuan, C. Grebogi, B. Hunt, E. Kostelich, E. Ott, and J. Yorke, "On the control of US Navy cranes," in Proc. ECC, Brussels, Belgium, July 1997.

[45] P. Martin, "Contribution à l'étude des systémes différentiellement plats,' Ph.D. dissertation, École des Mines, Paris, 1992.

[46] _ "An intrinsic sufficient condition for regular decoupling," Syst. Contr. Lett., vol. 20, pp. 383-391, 1993.

[47] _ _Endogenous feedbacks and equivalence," in Systems and Networks: Mathematical Theory and Applications, U. Helmke, R. Mennicken, and J. Saurer, Eds., Proc. MTNS-93, vol. 2. Berlin, Germany: Akademie Verlag, 1994, pp. 343-346.

[48] _ , "Aircraft control using flatness," in Proc. CESA, Lille, France, 1996, pp. 194-199.

[49] P. Martin, S. Devasia, and B. Paden, "A different look at output tracking: Control of a VTOL aircraft," Automatica, vol. 32, pp. 101-107, 1996.

[50] P. Martin, R. M. Murray, and P. Rouchon, "Flat systems," Plenary Lectures and Minicourses, G. Bastin and M. Gevers, Eds., Proc. ECC, Brussels, Belgium, 1997, pp. 211-264.

[51] P. Martin and P. Rouchon, "Feedback linearization and driftless systems," Math. Contr. Signal Syst., vol. 7, pp. 235-254, 1994.

[52] _ . "Any (controllable) driftless system with 3 inputs and 5 states is flat," Syst. Contr. Lett., vol. 25, pp. 167-173, 1995.

[53] _ "Flatness and sampling control of induction motors," in Proc. IFAC World Congr., San Francisco, CA, 1996.

[54] P. J. McLellan, "A differential-algebraic perspective on nonlinear controller design methodologies," Chemical Eng. Sci., vol. 49, pp. 1663-1679, 1994.

[55] P. Mullhaupt, B. Srinivasan, J. Lévine, and D. Bonvin, "A toy more difficult to control than the real thing," in Proc. ECC, Brussels, Belgium, July 1997.

[56] M. van Nieuwstadt and R. M. Murray, "Approximate trajectory generation for differentially flat systems with zero dynamics," in Proc. 34th IEEE Conf. Decision Control, New Orleans, LA, 1995, pp. 4224-4230. 
[57] M. van Nieuwstadt, M. Rathinam, and R. M. Murray, "Differential flatness and absolute equivalence," in Proc. 33th IEEE Conf. Decision Control, Lake Buena Vista, FL, 1994, pp. 326-333.

[58] H. Nijmeijer and A. J. van der Schaft, Nonlinear Dynamical Control Systems. New York: Springer, 1990.

[59] P. J. Olver, Applications of Lie Groups to Differential Equations. New York: Springer, 1986.

[60] _ Equivalence, Invariants, and Symmetry. Cambridge: Cambridge Univ. Press, 1995.

[61] J. B. Pomet, "A differential geometric setting for dynamic equivalence and dynamic linearization," in Geometry in Nonlinear Control and Differential Inclusions, B. Jakubczyk, W. Respondek, and T. Rzeżuchowski, Eds. Warsaw: Banach Center, 1995, pp. 319-339.

[62] _ "On dynamic feedback linearization of four-dimensional affine control systems with two inputs," ESAIM-COCV, 1997. Available http://www.etexinput.fr/Maths/Cocv/Articles/articleEng.html.

[63] M. Rathinam and R. M. Murray, "Configuration flatness of Lagrangian systems underactuated by one control," SIAM J. Contr. Optimiz., vol. 36, no. 1, pp. 164-179, 1998.

[64] R. Rothfuss, Anwendung der flachheitsbasierten Analyze und Regelung nichtlinearer Mehgrößensysteme, VDI, Düsseldorf, 1997.

[65] R. Rothfuss, J. Rudolph, and M. Zeitz, "Flatness based control of a nonlinear chemical reactor model," Automatica, vol. 32, pp. 1433-1439, 1996.

[66] P. Rouchon, "Necessary condition and genericity of dynamic feedback linearization,” J. Math. Systems Estim. Contr., vol. 4, pp. 257-260, 1994.

[67] P. Rouchon, M. Fliess, J. Lévine, and Ph. Martin, "Flatness, motion planning and trailer systems," in Proc. 32nd IEEE Conf. Decision and Control, San Antonio, TX, 1993, pp. 2700-2705.

[68] D. J. Saunders, The Geometry of Jet Bundles. Cambridge: Cambridge Univ. Press, 1989.

[69] W. F. Shadwick, "Absolute equivalence and dynamic feedback linearization,” Syst. Contr. Lett., vol. 15, pp. 35-39, 1990.

[70] A. J. van der Schaft, "Observability and controllability for smooth nonlinear systems," SIAM J. Contr. Optimiz., vol. 20, pp. 338-354, 1982

[71] W. M. Sluis, "Absolute equivalence and its application to control theory," Ph.D. dissertation, Univ. Waterloo, Ontario, Canada, 1992.

[72] "A necessary condition for dynamic feedback linearization," Syst. Contr. Lett., vol. 21, pp. 277-283, 1993.

[73] R. Sommer, "Control design for multivariable nonlinear time-varying systems," Int. J. Contr., vol. 31, pp. 883-891, 1980.

[74] H. J. Sussmann and V. Jurdjevic, "Controllability of nonlinear systems," J. Differential Equations, vol. 12, pp. 95-116, 1972.

[75] T. Tsujishita, "Formal geometry of systems of differential equations," Sugaku Expos., vol. 3, pp. 25-73, 1990.

[76] A. M. Vinogradov, "Local symmetries and conservation laws," Acta Appl. Math., vol. 2, pp. 21-78, 1984

[77] A. M. Vinogradov, Ed., Symmetries of Partial Differential Equations. Dordrecht: Kluwer, 1989.

[78] A. M. Vinogradov, "From symmetries of partial differential equations toward secondary ('quantized') calculus," J. Geometry Physics, vol. 14, pp. 146-194, 1994

[79] E. T. Whittaker, A Treatise on the Analytical Dynamics of Particles and Rigid Bodies, 4th ed. Cambridge: Cambridge Univ. Press, 1937.

[80] V. V. Zharinov, Geometrical Aspect of Partial Differential Equations. Singapore: World Scientific, 1992.

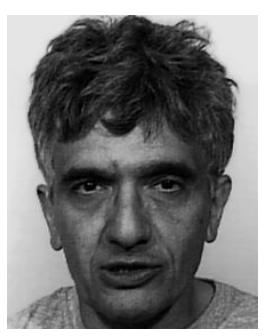

Michel Fliess graduated from the École Polytechnique, Paris. He obtained the Thèse de $3^{e}$ cycle from the Faculté des Sciences de Paris and the Thèse d'État from the Université Paris VII in 1969 and 1972.

From 1973 to 1983, he was a Professor at the Université Paris VIII. From 1983 to 1998, he was Directeur de Recherche au CNRS at the Laboratoire des Signaux et Systèmes, CNRS-Supélec-Université Paris-Sud. His current research interests include finite-dimensional nonlinear systems and infinite-dimensional linear systems.

Dr. Fliess was awarded the Michel Montpetit prize from the Paris Academy of Sciences in 1987 and the Silver Medal from the CNRS in 1991.

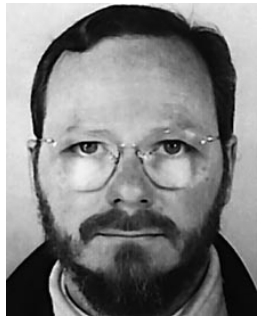

cesses.

Dr. Lévine is an Associate Editor of Mathematics of Control Signals and Systems.

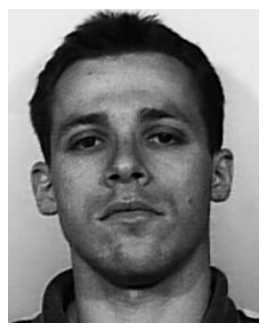

Philippe Martin received the Ph.D. degree in Mathematics and Control from the École des Mines de Paris in 1992, where he is currently a Research Associate. In 1993 and 1994, he visited the Center for Control Engineering and Computation of the University of California at Santa Barbara and the Department of Mathematics of the University of North Carolina, Chapel Hill. His research interests include theoretical aspects of nonlinear control and their applications to industrial control problems.

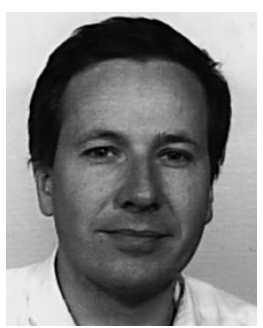

Pierre Rouchon was born in 1960 in Saint-Étienne, France. He graduated from "École Polytechnique" in 1983 and obtained the "Doctorat" in chemical engineering at École des Mines de Paris in 1990.

$\mathrm{He}$ is the Head of the Centre Automatique et Systèmes (Automatic Control Group) of École des Mines de Paris. His research interests include the theory and applications of nonlinear control, and in particular differential flatness and its extension to infinite-dimensional systems. He has worked on many industrial applications such as distillation columns, electrical drives, car equipments, and chemical reactors.

Dr. Rouchon is an Associate Editor of ESAIM-COCV, an electronic journal

Jean Lévine received the "Doctorat de 3ème cycle" rom the University Paris-Dauphine.

Systèmes (Systems and Control Center) of the École Mines de Paris since 1975. His research interest tillation columns, aircraft control, car equipments, chemical reactors and food and bioengineering pro.

然

